\title{
Critical review on the stability of illicit drugs in sewers and
}

\section{wastewater samples}

Ann-Kathrin McCall a ${ }^{\mathrm{a}}$, Richard Bade ${ }^{\mathrm{b}}$, Juliet Kinyua ${ }^{\mathrm{c}}$, Foon Yin Lai ${ }^{\mathrm{d}}$, Phong K. Thai ${ }^{\mathrm{d}, \mathrm{e}}$, Adrian Covaci $^{\mathrm{c}}$, Lubertus Bijlsma ${ }^{\mathrm{b}}$, Alexander L.N. van Nuijs ${ }^{\mathrm{c}}$, Christoph Ort ${ }^{\mathrm{a}, *}$

${ }^{a}$ Eawag, Swiss Federal Institute of Aquatic Science and Technology, CH 8600 Dübendorf,

Switzerland

${ }^{\mathrm{b}}$ Research Institute for Pesticides and Water, University Jaume I, Avda. Sos Baynat, E-12071 Castellón, Spain

${ }^{\mathrm{c}}$ Toxicological Centre, Department of Pharmaceutical Sciences, University of Antwerp (UA), Universiteitsplein 1, 2610 Antwerp, Belgium

${ }^{\mathrm{d}}$ The University of Queensland, The National Research Centre for Environmental Toxicology (Entox), 39 Kessels Rd., Coopers Plains, Brisbane, QLD 4108, Australia

${ }^{\mathrm{e}}$ Queensland University of Technology, International Laboratory for Air Quality \& Health, 2 George Street, Brisbane, QLD 4001, Australia 


\begin{abstract}
Wastewater-based epidemiology (WBE) applies advanced analytical methods to quantify drug residues in wastewater with the aim to estimate illicit drug use at the population level. Transformation processes during transport in sewers (chemical and biological reactors) and storage of wastewater samples before analysis are expected to change concentrations of different drugs to varying degrees. Ignoring transformation for drugs with low to medium stability will lead to an unknown degree of systematic under- or overestimation of drug use, which should be avoided. This review aims to summarize the current knowledge related to the stability of commonly investigated drugs and, furthermore, suggest a more effective approach to future experiments. From over 100 WBE studies, around 50 mentioned the importance of stability and 24 included tests in wastewater. Most focused on in-sample stability (i.e., sample preparation, preservation and storage) and some extrapolated to insewer stability (i.e., during transport in real sewers). While consistent results were reported for rather stable compounds (e.g., MDMA and methamphetamine), a varying range of stability under different or similar conditions was observed for other compounds (e.g., cocaine, amphetamine and morphine). Wastewater composition can vary considerably over time, and different conditions prevail in different sewer systems. In summary, this indicates that more systematic studies are needed to: i) cover the range of possible conditions in sewers and ii) compare results more objectively. To facilitate the latter, we propose a set of parameters that should be reported for in-sewer stability experiments (laboratory and full-scale). Finally, a best practice of sample collection, preservation, and preparation before analysis is suggested in order to minimize transformation during these steps.
\end{abstract}

45 Keywords: Transformation, sewage epidemiology, sample preservation, psychoactive substances, biodegradation. 


\section{Content}

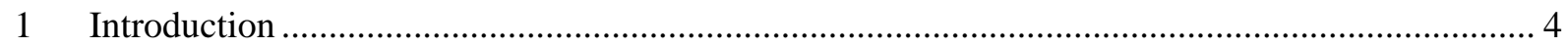

1.2 Stability of illicit drug biomarkers during wastewater treatment processes and in the environment.

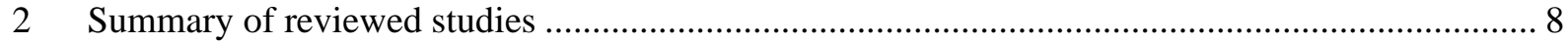

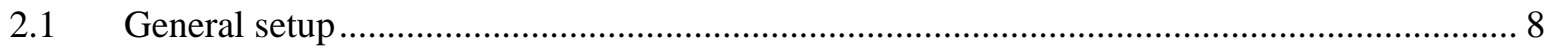

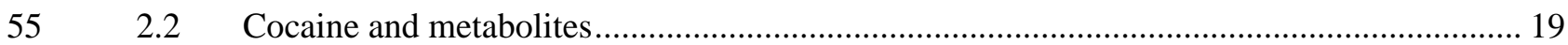

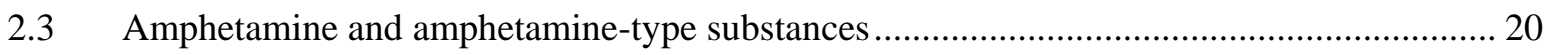

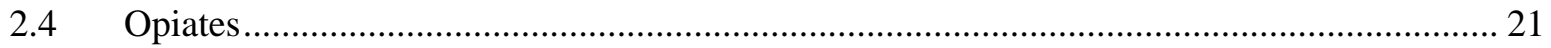

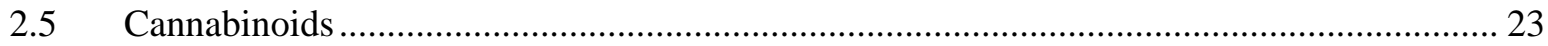

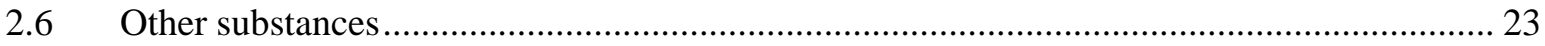

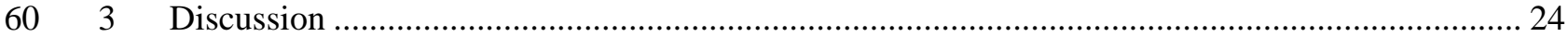

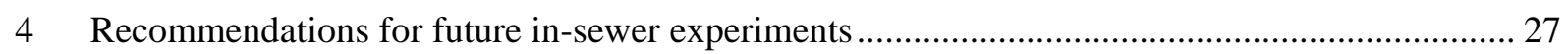

5 Recommendations for preserving illicit drugs in wastewater samples....................................... 30

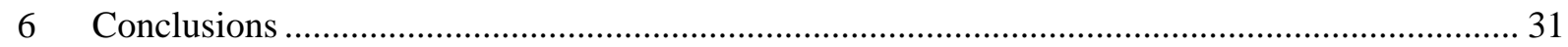

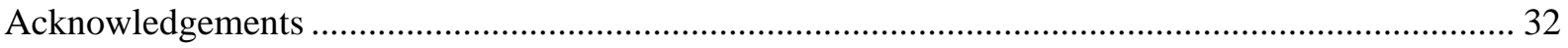



\section{Abbreviations}

6-MAM 6-monoacetyl morphine

$70 \quad \mathrm{BE}$

AMP Amphetamine

BE Benzoylecgonine

COC Cocaine

COCA Cocaethylene

COD Codeine

EDDP 2-ethylidene-1,5-dimethyl-3,3-diphenylpyrrolidine

75 EME Ecgonine methyl ester

KET Ketamine

LSD Lysergic acid diethylamide

MBDB Methylbenzodioxolylbutanamine

MDA 3,4-methylenedioxyamphetamine

80 MDEA

3,4-methylenedioxy-N-ethylamphetamine

MDMA 3,4-methylenedioxy-methamphetamine

METH Methamphetamine

MOR Morphine

MTD Methadone

85 nor-BE

Nor-benzoylecgonine

nor-COC Nor-cocaine

SPE Solid-phase extraction

SPM Suspended particulate matter

THC $\quad \Delta$ 9-tetrahydrocannabinol

90 THC-COOH

11-nor-9-carboxy-THC

THC-OH 11-hydroxy-THC

TSS

VSS

Total suspended solids

Volatile suspended solids

WBE Wastewater-based epidemiology 


\section{Introduction}

Wastewater-based epidemiology (WBE) is a recently introduced monitoring tool in drug epidemiology. It provides objective information about the levels and patterns of drug use at the population level and as such is complementary to existing survey-based methods. It has also the potential to serve as an early warning system for the use of new psychoactive substances (NPS, e.g., Kinyua et al., 2015; Reid et al., 2014) and to investigate the effectiveness of intervention programs (e.g., Burgard et al., 2014; Castiglioni et al., 2014). The principle of WBE is predicated by the fact that substances are excreted as parent compounds and/or metabolites - subsequently referred to as biomarkers (of illicit drugs) - after consumption and transported through the sewer network to wastewater treatment plants (Figure 1).

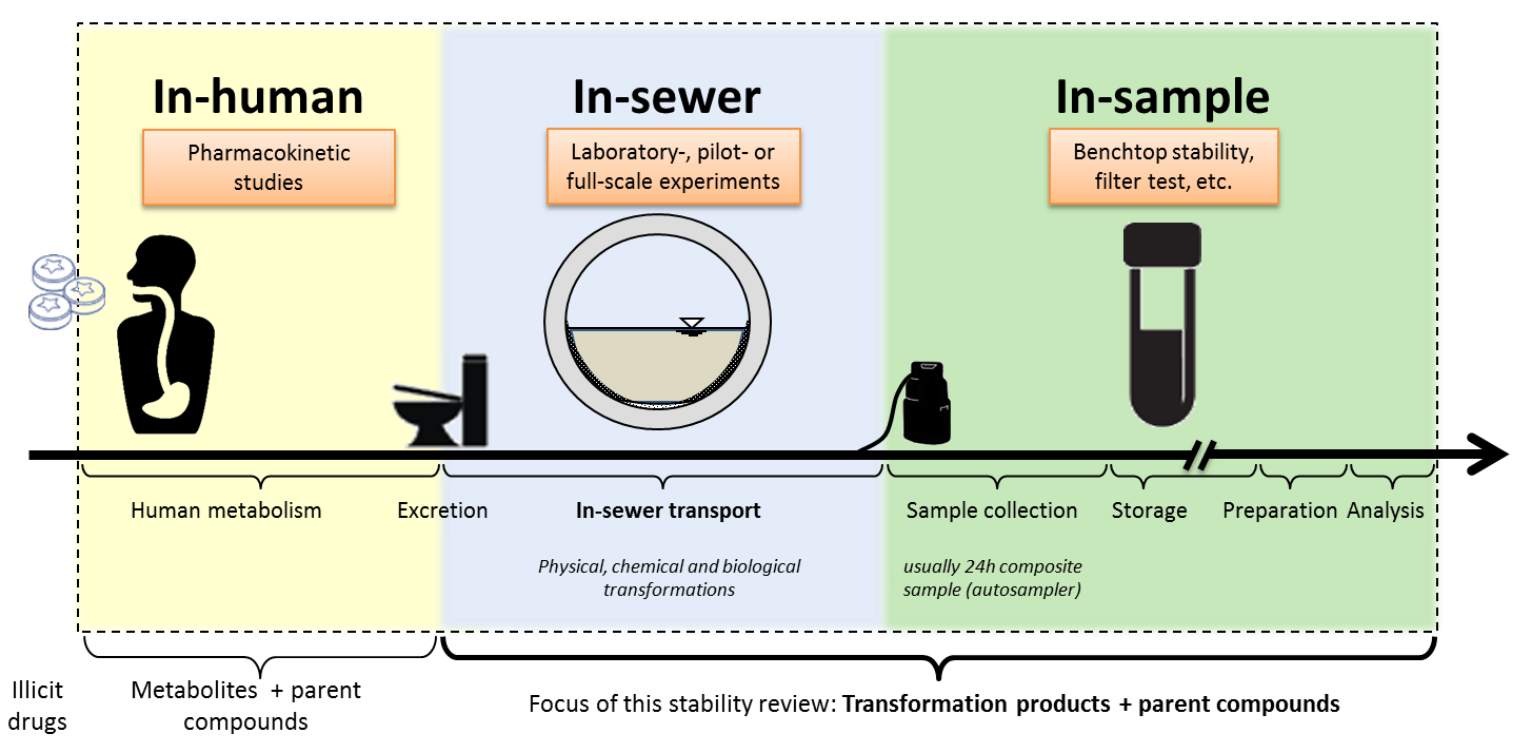

Figure 1. System boundaries used in wastewater-based epidemiology.

The concentrations of biomarkers in the wastewater $\left(c_{i}\right)$ can be quantified with advanced analytical instruments, such as liquid chromatography coupled to tandem mass spectrometry (LC-MS/MS). Consumption estimates are calculated according to Eq. 1:

$$
\operatorname{drug~use~}_{i}=\frac{Q \times C_{i} \times m_{i}}{P \times e_{i} \times p_{i}}
$$


where $Q=$ wastewater volume, $c_{i}=$ concentration of drug i, $m_{i}=$ molar mass ratio (parent to metabolite), $P=$ population for normalization, $e_{i}=$ drug-specific pharmacokinetic excretion rate 115 (average or distribution of urinary excretion, e.g., van Nuijs et al., 2011a; Zuccato et al., 2005) and $p_{i}$ $=$ purity of drug. In 2005, WBE was applied for the first time, back-calculating the cocaine use of communities in Italy (Zuccato et al., 2005). Since then, studies have compared temporal and spatial drug use trends by consumption differences between rural and metropolitan areas (e.g., Irvine et al., 2011; van Nuijs et al., 2009a; van Nuijs et al., 2011b), among different countries (e.g., Ort et al., 2014; Thomas et al., 2012) and during special events (festivals, holidays (e.g., Lai et al., 2013)) to name some of several applications.

Most illicit drugs may or may not have licit medical purposes, but they are produced, trafficked and/or consumed illegally on a large scale (United Nations Office of Drug and Crime, 2014). In this review, we focus on the most frequently used illicit drugs and their metabolites: cocaine, amphetamines and amphetamine-type substances, opiates, cannabinoids and selected other illicit substances, such as ketamine (KET) and lysergic acid diethylamide (LSD).

One of the main challenges in WBE is to reduce the uncertainty of each variable in the backcalculation equation (Eq. 1). High uncertainty is related to the excretion rates based on pharmacokinetic literature (Castiglioni et al., 2013) and the chemical analysis of the biomarkers in the complex wastewater matrix. Numerous efforts have focused on improving the accuracy (trueness and precision) of different analytical methods, and inter-laboratory studies were conducted in order to evaluate and harmonize the different analytical procedures being used (Castiglioni et al., 2013; Thomas et al., 2012). Uncertainties associated with the population estimates have recently been conceptually reduced (Lai et al., 2015; O'Brien et al., 2014), as well as the uncertainty related to wastewater sampling (Ort et al., 2010). Furthermore, sample collection, storage and preparation methods have been evaluated, deducing critical, substance-specific parameters: i) solvent and temperature used during the evaporation of solid-phase extraction (SPE) extracts, and ii) biomarker stability in the wastewater matrix and silanisation of glassware (Baker and Kasprzyk-Hordern 2011a). To further minimize uncertainty of WBE, a better understanding of in-sewer and in-sample stability of biomarkers is needed (Fig. 1). 
Castiglioni et al. (2013) estimated that the uncertainty related to the stability of the biomarkers during in-sewer transport is less than $10 \%$. However, they also concluded that more research is needed. Other studies urge the consideration of the stability of the illicit drug biomarkers in the back-calculation method (van Nuijs et al., 2009b; Östman et al., 2014).

While in-sample stability has been studied to some degree for most of the biomarkers (e.g., Baker and Kasprzyk-Hordern, 2011a; Chen et al., 2013; Östman et al., 2014), their in-sewer stability under the influence of varying environmental conditions is not well understood. The sewer is considered a biological and chemical reactor, influenced by physical processes (Hvitved-Jacobsen et al., 2013). Residence times of $30 \mathrm{~min}$ to $12 \mathrm{~h}$ (rarely up to $24 \mathrm{~h}$ ) in most catchments and potential environmental processes facilitate formation of transformation products (Heuett et al., 2015a). Consequently, the omission of biomarker-specific in-sewer transformation may add an unknown level of uncertainty. Yet, only few studies have investigated in-sewer stability of selected biomarkers under environmental conditions (Senta et al., 2014; Thai et al., 2014; van Nuijs et al., 2012) and accounted for stability in the back-calculation of drug use (Baker et al., 2014; Östman et al., 2014).

In this review, we summarize and critically evaluate the available scientific literature focusing on the stability of the most frequently used illicit drugs during i) in-sewer transport and ii) in-sample storage. Using this information, more insight is obtained regarding the uncertainty of WBE associated with stability and, additionally, suggestions for best practices in future stability studies are provided.

\subsection{Environmental processes in sewer networks}

In general, two major categories of processes determine the overall fate of illicit drug biomarkers in the sewer network. First, mass transfer processes that leave the structure of the chemicals unchanged, e.g., transport, mixing and transfer among different phases and/or compartments (sorption, sedimentation, and uptake by organisms). The second category includes processes that alter the structure of the compounds, e.g., chemical and/or biological transformation reactions (Schwarzenbach et al., 2003b). For the remainder of the manuscript, the term transformation will be used to refer to any of these three processes, although physical processes are rather transfer, not transformation, processes. 
Wastewater contains a large number of soluble, colloidal, and suspended components (e.g., nutrients, metals, micropollutants and pathogenic and nonpathogenic microorganisms). Its content varies in time and space, which favors or inhibits specific environmental processes. In addition, sewer designs and operation modes influence the prevailing conditions, e.g., oxygen concentrations (redox potential), $\mathrm{pH}$, temperature, flow velocities and sediments (Figure 2). The dominating processes that may influence biomarker concentrations in the sewer are most likely residence time, abiotic and biotic transformations (e.g., hydrolysis, deconjugation, biodegradation), as well as sorption to SPM. Further, the presence of biofilms on the sewer walls should be accounted for (Hvitved-Jacobsen et al., 2013).

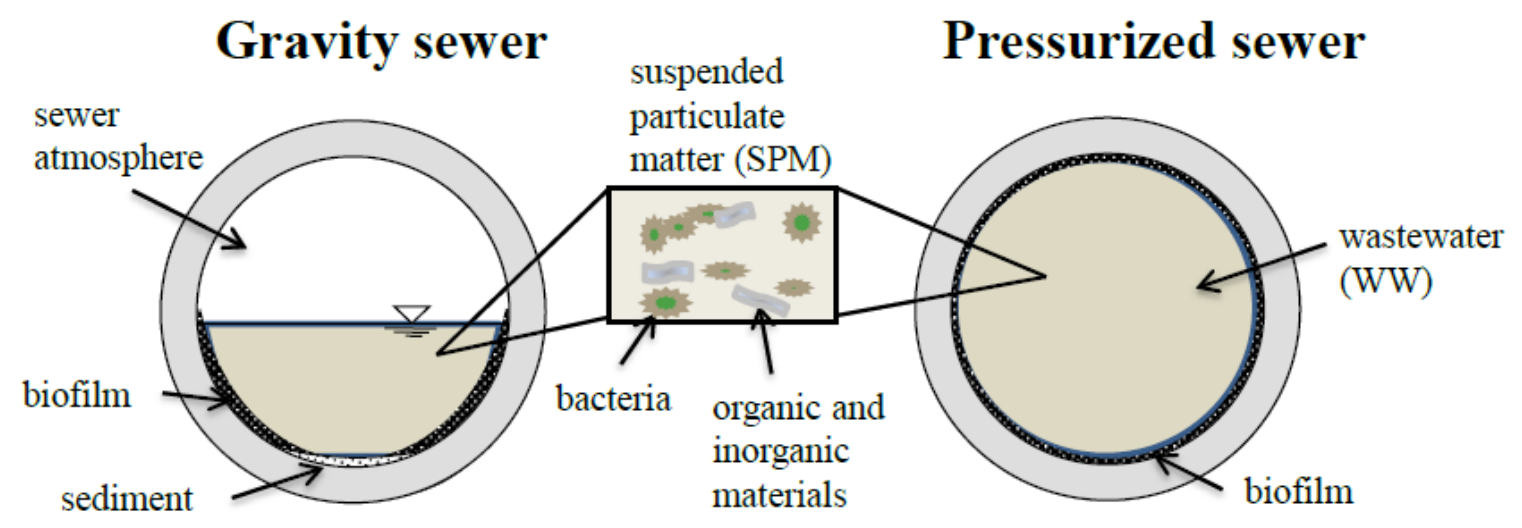

Figure 2. Cross-sections of gravity driven and pressurized sewers.

\subsection{Stability of illicit drug biomarkers during wastewater treatment processes and in the environment}

180 It is noteworthy that compounds may also be transformed through numerous processes, e.g., chlorination or ozonation, during wastewater treatment or photodegradation in the environment. Effects of these processes and resulting transformation products were beyond the scope of this review. To fully assess their fate, transport and toxicological impact on the natural environment, comprehensive monitoring and additional investigations are needed (Bijlsma et al., 2013; Heuett et al., 2015a). Several reviews are available for these pertinent topics (Bijlsma et al., 2013; Boix et al., 2014; Postigo et al., 2011a, 2011b; Rodayan et al., 2014; Heuett et al., 2015a). 


\section{Summary of reviewed studies}

More than 50 WBE studies mentioned the importance of stability, from which 24 actually investigated in-sewer or in-sample stability to some extent. Overall, a clear distinction between in-sewer and insample stability is lacking in the current literature.

\subsection{General setup}

In-sewer stability experiments should account for all relevant processes occurring in sewer compartments: i) the bulk liquid (wastewater with suspended particulate matter (SPM)), ii) the biofilm growing on the sewer walls, iii) the sediments, and iv) the sewer atmosphere in gravity sewers. Most studies only investigated the stability in the bulk liquid. No experiments to date have investigated the effect of sewer sediments or the sewer atmosphere on biomarker transformation. Focusing on illicit drugs, only one pilot-scale sewer reactor study included the sewer wall biofilm (Thai et al., 2014). Considering the physico-chemical properties of most biomarkers (Table S1) and their hydrophilic character, precipitation and evaporation seem negligible (Ternes and Joss, 2006).

In this review, we consider all laboratory studies using unfiltered wastewater at a typical $\mathrm{pH}$ around 78 , at temperatures above $10^{\circ} \mathrm{C}$ as in-sewer studies. Nonetheless, the experimental setups of these studies differed in complexity with different degrees of approximation. Several research groups conducted stability studies as part of a validation method, and only a few publications showcased an exclusive focus on analyte stability in wastewater. Consequently, the main study conditions are summarized in Table 1. Usually, the concentration of biomarkers in spiked, unfiltered wastewater was monitored in glass or plastic containers, in the dark, under constant $\mathrm{pH}$ and temperature, over periods of $12-72 \mathrm{~h}$. Six publications considered the fraction of biomarker that bound to SPM or sludge (in wastewater treatment plants). 
Table 1. Overview of experimental conditions

\begin{tabular}{|c|c|c|c|c|c|c|c|c|c|c|c|}
\hline 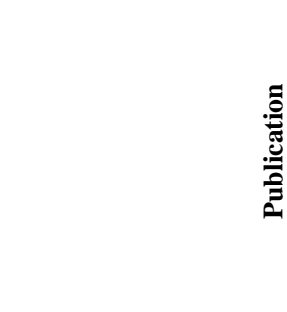 & 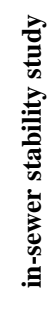 & 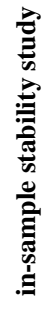 &  & 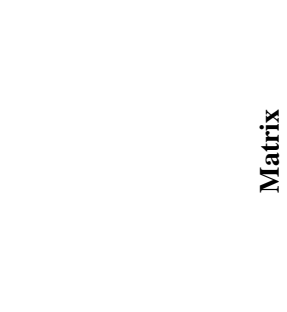 & 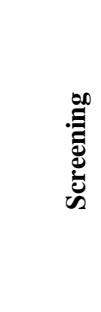 & 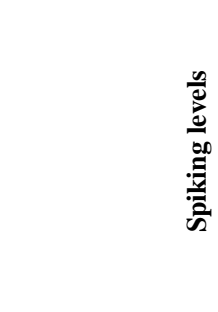 & 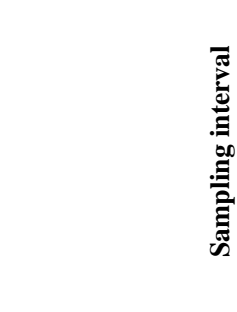 & 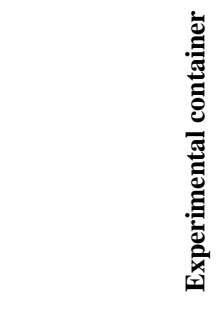 & Th & 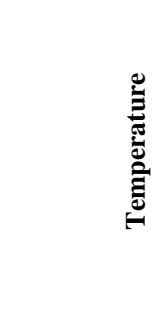 & 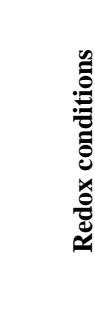 \\
\hline \multirow{3}{*}{$\begin{array}{l}\text { Baker and Kasprzyk- } \\
\text { Hordern, 2011a }\end{array}$} & $\mathrm{x}$ & & & unfiltered WW & \multirow{3}{*}{ target } & $1 \mu \mathrm{g} / \mathrm{L}$ & $0,12,24,72 \mathrm{~h}$ & \multirow{3}{*}{$\begin{array}{l}\text { amber silanized } \\
\text { glass bottles }\end{array}$} & $\mathrm{pH} 7.4$ & $19^{\circ} \mathrm{C}$ & \multirow{3}{*}{ NA } \\
\hline & & $\mathrm{x}$ & & $\begin{array}{l}\text { unfiltered and filtered } \\
\text { WW } \\
\end{array}$ & & $1 \mu \mathrm{g} / \mathrm{L}$ & $0,12,24,72 \mathrm{~h}$ & & $\mathrm{pH} 7.4$ and 1.8 & $\begin{array}{c}2^{\circ} \mathrm{C} \text { and } \\
19^{\circ} \mathrm{C}\end{array}$ & \\
\hline & & $\mathrm{x}^{\mathrm{a}}$ & & $\begin{array}{c}\text { filtered WW (filter type } \\
\text { not reported) }\end{array}$ & & $1 \mu \mathrm{g} / \mathrm{L}$ & $0,2,4,6$ weeks & & $\mathrm{pH} 2$ & $-20^{\circ} \mathrm{C}$ & \\
\hline $\begin{array}{l}\text { Baker and Kasprzyk- } \\
\text { Hordern, 2011b }\end{array}$ & & & $\mathrm{x}$ & unfiltered WW & target & none & not relevant & $\begin{array}{l}\text { amber silanised } \\
\text { bottles }\end{array}$ & NA & NA & NA \\
\hline $\begin{array}{c}\text { Bisceglia and Lippa, } \\
2014\end{array}$ & $\mathrm{x}$ & & & $\begin{array}{l}\text { WW (coarsely filtered } \\
11 \mu \mathrm{m} \text { Whatman Nb1) }\end{array}$ & target & $\begin{array}{c}\text { 3-600x the } \\
\text { background } \\
\text { concentrations }\end{array}$ & $>10 \mathrm{x}$ over $24 \mathrm{~h}$ & $\begin{array}{c}\text { 1-L glass } \\
\text { Erlenmeyer flask }\end{array}$ & $\mathrm{pH} 7.3$ & $\begin{array}{c}9^{\circ} \mathrm{C}, 23^{\circ} \mathrm{C} \\
31^{\circ} \mathrm{C}\end{array}$ & NA \\
\hline Boix et al., 2014 & & $\mathrm{x}$ & & WW, surface water & suspect & $1000 \mu \mathrm{g} / \mathrm{L}$ & $\begin{array}{c}0,1,3,7,10,17 \\
\text { days }\end{array}$ & NA & NA & $\begin{array}{c}\text { room } \\
\text { temperature }\end{array}$ & NA \\
\hline Burgard et al., 2013 & & $\mathrm{x}$ & & unfiltered WW & target & $1 \mu \mathrm{g} / \mathrm{L}$ & $8 \mathrm{x}$ over $72 \mathrm{~h}$ & glass container & $\mathrm{NA}$ & $\max .20^{\circ} \mathrm{C}$ & $\mathrm{NA}$ \\
\hline Castiglioni et al., 2011a & & $\mathrm{x}$ & & unfiltered WW & target & $1-5 \mu \mathrm{g} / \mathrm{L}$ & $\begin{array}{c}0,1,3 \text { days; } 3 \\
\text { freeze-thaw cycles }\end{array}$ & glass bottles & NA & $\begin{array}{c}4^{\circ} \mathrm{C},-20^{\circ} \mathrm{C} \\
\text { freeze-and- } \\
\text { thaw }\end{array}$ & NA \\
\hline Castiglioni et al., 2006 & & $\mathrm{x}$ & & unfiltered WW & target & $0.5-5 \mu \mathrm{g} / \mathrm{L}$ & 0,3 days & glass bottles & NA & $4^{\circ} \mathrm{C}$ & NA \\
\hline \multirow{2}{*}{ Castiglioni et al., 2015} & & $\mathrm{x}$ & & unfiltered WW & \multirow{2}{*}{ target } & $0.1 \mu \mathrm{g} / \mathrm{L}$ & $0,3,6,24,48 \mathrm{~h}$ & glass bottles & NA & $\begin{array}{l}4^{\circ} \mathrm{C} ; \text { room } \\
\text { temperature }\end{array}$ & NA \\
\hline & $\mathrm{x}$ & & & unfiltered WW & & $0.1 \mu \mathrm{g} / \mathrm{L}$ & $0,3,6,24,48 \mathrm{~h}$ & glass bottles & NA & $\begin{array}{c}\text { room } \\
\text { temperature }\end{array}$ & NA \\
\hline
\end{tabular}




\begin{tabular}{|c|c|c|c|c|c|c|c|c|c|c|c|}
\hline \multirow{2}{*}{ Chen et al., 2013} & $\mathrm{x}$ & & & unfiltered WW & \multirow{2}{*}{ target } & $>0.1 \mu \mathrm{g} / \mathrm{L}$ & $0,1,2,3,7,14$ days & NA & NA & $20^{\circ} \mathrm{C}$ & NA \\
\hline & & $\mathrm{x}$ & & $\begin{array}{c}\text { unfiltered WW, filtered } \\
\text { WW, unfiltered WW } \\
+\mathrm{Na}_{2} \mathrm{~S}_{2} \mathrm{O}_{5}\end{array}$ & & $>0.1 \mu \mathrm{g} / \mathrm{L}$ & $0,1,2,3,7,14$ days & NA & $\begin{array}{l}\mathrm{pH} \text { of } \mathrm{WW}(\sim 7) \\
\text { and at } \mathrm{pH} 2\end{array}$ & $\begin{array}{l}20^{\circ} \mathrm{C}, 4^{\circ} \mathrm{C} \\
\text { and }-20^{\circ} \mathrm{C}\end{array}$ & NA \\
\hline Chiaia et al., 2008 & & $\mathrm{x}$ & & unfiltered WW & target & $0.2 \mu \mathrm{g} / \mathrm{L}$ & $\begin{array}{c}1,2,3,4,7,14,21 \\
\text { days }\end{array}$ & HDPE bottles & $\begin{array}{l}\mathrm{pH} \text { of } \mathrm{WW}(\sim 7) \\
\text { and at } \mathrm{pH} 2\end{array}$ & $\begin{array}{c}\text { room } \\
\text { temperature, } \\
4^{\circ} \mathrm{C},-20^{\circ} \mathrm{C}\end{array}$ & NA \\
\hline Gheorghe et al., 2008 & & $\mathrm{x}$ & & surface water & target & $0.1-0.4 \mu \mathrm{g} / \mathrm{L}$ & $0,1,3,5$ days & glass bottles & $\mathrm{pH} 6$ and 2 & $\begin{array}{c}-20^{\circ} \mathrm{C}, 4^{\circ} \mathrm{C} \\
\text { and } 20^{\circ} \mathrm{C}\end{array}$ & NA \\
\hline $\begin{array}{l}\text { González-Mariño et al., } \\
2010\end{array}$ & & $\mathrm{x}$ & & $\begin{array}{l}\text { filtered } \mathrm{WW} \text { and filtered } \\
\qquad \mathrm{WW}+\mathrm{NaN}_{3}\end{array}$ & target & $100 \mu \mathrm{g} / \mathrm{L}$ & $\begin{array}{c}0,1,3,5,7,14,21 \\
84 \text { days }\end{array}$ & $\begin{array}{l}\text { amber glass } \\
\text { bottles }\end{array}$ & NA & $\begin{array}{l}4^{\circ} \mathrm{C} \text { and - } \\
20^{\circ} \mathrm{C}\end{array}$ & NA \\
\hline Heuett et al., 2015b & & $\mathrm{x}$ & & unfiltered WW & target & $0.25 \mu \mathrm{g} / \mathrm{L}$ & $\begin{array}{c}0,3,7,17,27, \text { and } \\
123 \text { days }\end{array}$ & glass bottles & NA & $-20^{\circ} \mathrm{C}$ & NA \\
\hline Jelic et al., 2014 & $\mathrm{x}$ & & & $\begin{array}{c}\text { unfiltered WW } \\
+ \text { +sewerwall biofilm }\end{array}$ & target & no spike & $0,21 \mathrm{~h}$ & NA (real sewer) & $\mathrm{pH} 7.2-7.6$ & $22^{\circ} \mathrm{C}$ & anaerobic \\
\hline Langford et al., 2011 & & & $\mathrm{x}$ & sludge & target & no spike & not relevant & $\begin{array}{l}\text { silanized glass } \\
\text { flask }\end{array}$ & NA & NA & NA \\
\hline Mardal and Meyer, 2014 & $x^{b}$ & & & $\begin{array}{l}\text { unfiltered WW, } \\
\text { unfiltered WW +rat urine } \\
\text { and feces }\end{array}$ & suspect & $100 \mu \mathrm{g} / \mathrm{L}$ & $\begin{array}{c}0,1,2,3,4,5,6,7 \\
\text { days }\end{array}$ & $\begin{array}{l}\text { amber glass } \\
\text { bottles }\end{array}$ & NA & $22^{\circ} \mathrm{C}$ & aerobic \\
\hline Metcalfe et al., 2010 & & & $\mathrm{x}$ & unfiltered WW & suspect & none & not relevant & NA & $\mathrm{pH} 3$ & NA & NA \\
\hline Östman et al., 2014 & & $\mathrm{x}$ & & $\begin{array}{l}\text { filtered WW; purified } \\
\text { water }\end{array}$ & target & $0.9 \mu \mathrm{g} / \mathrm{L}$ & $0,24 \mathrm{~h}$ & $\begin{array}{c}\text { polyethylene } \\
\text { bottles (HDPE) }\end{array}$ & NA & $\begin{array}{c}\text { room } \\
\text { temperature; } \\
4^{\circ} \mathrm{C}\end{array}$ & NA \\
\hline
\end{tabular}




\begin{tabular}{|c|c|c|c|c|c|c|c|c|c|c|c|}
\hline \multirow[t]{2}{*}{ Plósz et al., 2013} & $\mathrm{x}$ & & & $\begin{array}{l}\text { unfiltered WW + } \\
\text { activated sludge }\end{array}$ & \multirow[t]{2}{*}{ target } & no spike & $\begin{array}{c}0,15,30,60,90 \\
120 \text { min, } 4,6,8,10 \\
16,18 \text { and } 24 \mathrm{~h}\end{array}$ & $\begin{array}{l}\text { glass reaction } \\
\text { vessel }\end{array}$ & $\mathrm{pH} 7.4$ & $21^{\circ} \mathrm{C}$ & $\begin{array}{c}\text { aerobic } \\
\text { and } \\
\text { anaerobic }\end{array}$ \\
\hline & & & $\mathrm{x}$ & $\begin{array}{c}\text { preclarified WW } \\
\text { +mercury chloride }\end{array}$ & & no spike & $\begin{array}{c}0,10,20,30,45,60 \\
\min \end{array}$ & $\begin{array}{l}\text { glass reaction } \\
\text { vessel }\end{array}$ & $\mathrm{pH} 7.4$ & NA & NA \\
\hline Rosa Boleda et al., 2011 & & $\mathrm{x}$ & & drinking water & target & none & $0,3,5,8$ days & $\begin{array}{c}\text { sterilized } \\
\text { polypropylene } \\
\text { bottles with } \\
\text { sodium thiosulfate }\end{array}$ & NA & $\begin{array}{l}4^{\circ} \mathrm{C} \text { and } \\
\text { room } \\
\text { temperature }\end{array}$ & NA \\
\hline \multirow{3}{*}{ Senta et al., 2014} & $\mathrm{x}$ & & & unfiltered WW & \multirow{3}{*}{ target } & $\begin{array}{c}0.2 \mu \mathrm{g} / \mathrm{L} \\
\text { cannabinioids } 1 \\
\text { ug/L }\end{array}$ & $0,4,6,24,48,72 \mathrm{~h}$ & glass bottles & $\mathrm{pH} 7.5$ & $10^{\circ}$ and $20^{\circ}$ & NA \\
\hline & & & $\mathrm{x}$ & unfiltered WW & & $4 \mu \mathrm{g} / \mathrm{L}$ & $0,4,6,24,48,72 \mathrm{~h}$ & glass bottles & $\mathrm{pH} 7.5$ & $10^{\circ}$ and $20^{\circ}$ & NA \\
\hline & & $\mathrm{x}$ & & unfiltered WW & & $0.25 \mu \mathrm{g} / \mathrm{L}$ & $0,24 \mathrm{~h}$ & $\begin{array}{l}\text { HD polypropylene } \\
\text { bottles }\end{array}$ & $\mathrm{pH} 7.5$ and 2 & $4^{\circ} \mathrm{C}$ & NA \\
\hline Senta et al., 2013 & & & $\mathrm{x}$ & $\begin{array}{l}\text { unfiltered raw WW, } \\
\text { secundary effluent WW, } \\
\text { activated sludge }\end{array}$ & target & none & not relevant & NA & $\mathrm{pH} \approx 7.5$ & NA & NA \\
\hline $\begin{array}{l}\text { Subedi and Kannan, } \\
2014\end{array}$ & & & $\mathrm{x}$ & $\begin{array}{l}\text { unfiltered raw WW, } \\
\text { primary effluent WW, } \\
\text { secundary effluent WW, } \\
\text { activated sludge, sludge }\end{array}$ & target & none & not relevant & amber glass jars & NA & NA & NA \\
\hline Thai et al., 2014 & $\mathrm{x}$ & & & $\begin{array}{l}\text { unfiltered WW in sewer } \\
\text { pilot reactor with } \\
\text { sewerwall biofilm }\end{array}$ & target & $\begin{array}{l}\text { deuterium labelled } \\
\text { substances } 10 \mu \mathrm{g} / \mathrm{L} \text {; }\end{array}$ & $\begin{array}{c}0,0.25,0.5,1,2,3 \\
\quad 6,9 \text { and } 12 \mathrm{~h}\end{array}$ & Perspex $^{\mathrm{TM}}$ & $\mathrm{pH} 7.5$ & $20^{\circ} \mathrm{C}$ & $\begin{array}{c}\text { gravity } \\
\text { sewer } \\
\text { (aerobic) } \\
\text { and rising } \\
\text { main } \\
\text { sewer } \\
\text { (anaerobic) }\end{array}$ \\
\hline van Nuijs et al., 2012 & $\mathrm{x}$ & & & unfiltered WW & target & $0.12-1.6 \mathrm{ug} / \mathrm{L}$ & $\begin{array}{c}0,1,2,3,4,5,6,7 \\
8,9,10,11,12,26 \mathrm{~h}\end{array}$ & $\begin{array}{l}\text { silanized glass } \\
\text { flask }\end{array}$ & $\mathrm{pH} 7.5$ & $20^{\circ} \mathrm{C}$ & NA \\
\hline
\end{tabular}




\begin{tabular}{|c|c|c|c|c|c|c|c|c|c|}
\hline Wick et al., 2011 & $(\mathrm{x})^{\mathrm{c}}$ & $\begin{array}{c}\text { unfiltered WW+activated } \\
\text { sludge }\end{array}$ & suspect & $2 \mu \mathrm{g} / \mathrm{L}$ & $8 \mathrm{x}$ over 3 days & $\begin{array}{l}\text { amber glass } \\
\text { bottles }\end{array}$ & $\begin{array}{c}\text { regulated at } \mathrm{pH} \\
7( \pm 0.2)\end{array}$ & NA & aerobic \\
\hline
\end{tabular}

${ }^{a}$ longterm stability study; ${ }^{b}$ incubation of WW with rat urine and feces to mimick human excreation; ${ }^{\mathrm{c}}$ Matrix was enriched with activated sludge to mimick the wastewater 
For most biomarkers, we found a range of transformation values, most likely as a result of different environmental conditions that were tested. Therefore, we propose to rate in-sample stability and insewer stability separately for each substance based on the available literature and our judgment: low 215 stability (60-100\% transformation), medium stability (20-60\% transformation), high stability (0-20\% transformation), or variable stability over $24 \mathrm{~h}$. The knowledge for the main groups of compounds is described in the subsequent sections. For other compounds and further information, see Table 2. 
Table 2. Literature summary of stability of illicit drug biomarkers from in-sewer and in-wastewater samples.

\begin{tabular}{|c|c|c|c|c|c|}
\hline \multicolumn{3}{|c|}{ In-sewer stability } & \multicolumn{3}{|c|}{ WW $=$ wastewater $*$ Substances used for consumption back-calculation } \\
\hline \multicolumn{3}{|c|}{ In-sample stability } & \multicolumn{3}{|c|}{$\begin{array}{l}\text { The stability of each substance during in-sewer transport and in-sample is rated as: stability is low (60-100\% transformation), } \\
\text { medium (20-60\% transformation), high (0-20\% transformation) or variable (if not otherwise indicated over } \mathbf{2 4 h} \text { ). }\end{array}$} \\
\hline 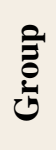 & Parent & Metabolite & 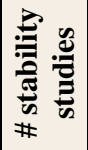 & Stability & References \\
\hline \multirow{7}{*}{ 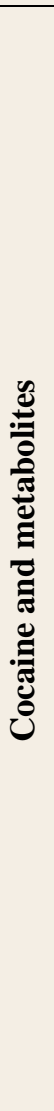 } & \multirow[b]{2}{*}{ Cocaine (COC) } & & 10 & $\begin{array}{l}\text { Low: } 60 \% \text { transformation over } 12 \mathrm{~h} @ 20^{\circ} \mathrm{C} \text { in gravity sewer laboratory } \\
\text { reactor -- } 100 \% \text { transformation expected over } 24 \mathrm{~h} \text {. In raising main } \\
\text { laboratory reactor } 45 \% \text { transformation occured over } 12 \mathrm{~h} \text {; with activated } \\
\text { sludge } 100 \% \text { transformation over } 24 \mathrm{~h} \text { at } 21^{\circ} \mathrm{C} \text { and } \mathrm{pH} 7.4\end{array}$ & $\begin{array}{c}\text { Baker and Kasprzyk-Hordern, 2011a; Bisceglia and } \\
\text { Lippa, 2014; Chen et al., 2013; Plósz et al., 2013; } \\
\text { Senta et al., 2013, 2014; Subedi and Kannan, 2014; } \\
\text { Thai et al., 2014; van Nuijs et al., 2012; Wick et al., } \\
\text { 2011 }\end{array}$ \\
\hline & & & 12 & $\begin{array}{l}\text { Low: at neutral pH: at } 4^{\circ} \mathrm{C}, 9^{\circ} \mathrm{C}, 19^{\circ} \mathrm{C}, 20^{\circ} \mathrm{C}, 23^{\circ} \mathrm{C} \& 31^{\circ} \mathrm{C} \text { over } 1-3 \mathrm{~d} \text {; } \\
\text { filtered/unfiltered WW at } 2^{\circ} \mathrm{C} \& 19^{\circ} \mathrm{C} \text { over } 3 \mathrm{~d} \text { (slightly higher for } \\
\text { unfiltered WW at } 20^{\circ} \mathrm{C} \text { over } 3 \mathrm{~d} \text { but lower afterwards); after two freeze- } \\
\text { and-thaw cycles within } 3 \mathrm{~d} \text {. High: neutral pH: at }-20^{\circ} \mathrm{C} \text { over } 3 \text { weeks; at } \\
2^{\circ} \mathrm{C} \text { over } 3 \mathrm{~d} \text {; low } p H \text { : at } 2^{\circ} \mathrm{C} \& 19^{\circ} \mathrm{C}-20^{\circ} \mathrm{C} \text { over } 3 \mathrm{~d} \text {; at }-20^{\circ} \mathrm{C} \text { over } 3 \\
\text { weeks; at } 37^{\circ} \mathrm{C} \text { over } 3.5 \mathrm{~h} \text {; in Milli-Q water at } 4^{\circ} \mathrm{C} \& 25^{\circ} \mathrm{C} \text { for } 24 \mathrm{~h} \text {; with } \\
\mathrm{Na}_{2} \mathrm{~S}_{2} \mathrm{O}_{2} \text { at } 20^{\circ} \mathrm{C} \text { for } 2 \text { weeks; absorbed on SPE cartridges (HLB) over } 12 \\
\text { weeks }\end{array}$ & $\begin{array}{l}\text { Baker and Kasprzyk-Hordern, 2011a, 2011b; } \\
\text { Castiglioni et al., 2006, 2011a; Chen et al., 2013; } \\
\text { Chiaia et al., 2008; Gheorghe et al., 2008; González- } \\
\text { Mariño et al., 2010; Heuett et al., 2015b; Metcalfe } \\
\text { etal., 2010; Östman et al., 2014; Senta et al., } 2014\end{array}$ \\
\hline & & \multirow[b]{2}{*}{$\begin{array}{l}\text { benzoylecgonine* } \\
\text { (BE) }\end{array}$} & 10 & $\begin{array}{l}\text { Medium: } 14 \% \text { transformation over } 12 \mathrm{~h} @ 20^{\circ} \mathrm{C} \text { in gravity sewer } \\
\text { laboratory reactor --> ca. } 28 \% \text { transformation expected over } 24 \mathrm{~h} \text {. In raising } \\
\text { main sewer } 8 \% \text { transformation occured over } 12 \mathrm{~h} \text {; with activated sludge } \\
80 \% \text { transformation over } 24 \mathrm{~h} \text { at } 21^{\circ} \mathrm{C} \text { and } \mathrm{pH} 7.4\end{array}$ & $\begin{array}{l}\text { Baker and Kasprzyk-Hordern, 2011a; Bisceglia and } \\
\text { Lippa, 2014; Chen et al., 2013; Plósz et al., 2013; } \\
\text { Senta et al., 2013, 2014; Subedi and Kannan, 2014; } \\
\text { Thai et al., 2014; van Nuijs et al., 2012; Wick et al., } \\
\text { 2011 }\end{array}$ \\
\hline & & & 12 & $\begin{array}{l}\text { High: at low pH: at } 2^{\circ} \mathrm{C} \& 19^{\circ} \mathrm{C}-20^{\circ} \mathrm{C} \text { over } 3 \mathrm{~d} \text {; at }-20^{\circ} \mathrm{C} \text { over } 3 \text { weeks; at } \\
37^{\circ} \mathrm{C} \text { over } 3.5 \mathrm{~h} \text {; at neutral pH: at }-20^{\circ} \mathrm{C} \text { over } 3 \text { weeks; at } 2^{\circ} \mathrm{C}, 4^{\circ} \mathrm{C}, 9^{\circ} \mathrm{C} \text {, } \\
19^{\circ} \mathrm{C}, 20^{\circ} \mathrm{C}, 23^{\circ} \mathrm{C} \& 31^{\circ} \mathrm{C} \text { over } 1-3 \mathrm{~d}, 7 \mathrm{~d} \text {; filtered / unfiltered WW at } 2^{\circ} \mathrm{C} \\
\& 19^{\circ} \mathrm{C} \text { over } 3 \mathrm{~d} \text { (higher for filtered WW at } 20^{\circ} \mathrm{C} \text { over } 14 \mathrm{~d} \text { ); in Milli-Q } \\
\text { water at } 4^{\circ} \mathrm{C} \& 25^{\circ} \mathrm{C} \text { for } 24 \mathrm{~h} \text {; after two freeze-and-thaw cycles within } 3 \mathrm{~d} \text {; } \\
\text { with } \mathrm{Na}_{2} \mathrm{~S}_{2} \mathrm{O}_{2} \text { at } 20^{\circ} \mathrm{C} \text { for } 2 \text { weeks; absorbed on SPE cartridges (HLB) over } \\
12 \text { weeks }\end{array}$ & $\begin{array}{l}\text { Baker and Kasprzyk-Hordern, 2011a; Castiglioni et } \\
\text { al., 2006, 2011a; Chen et al., 2013; Chiaia et al., 2008; } \\
\text { Gheorghe et al., 2008; González-Mariño et al., 2010; } \\
\text { Heuett et al., 2015b; Metcalfe etal., 2010; Östman et } \\
\text { al., 2014; Senta et al., } 2014\end{array}$ \\
\hline & & \multirow{2}{*}{$\begin{array}{l}\text { ecgonine methyl } \\
\text { ester (EME) }\end{array}$} & 3 & $\begin{array}{c}\text { Medium: } 20-40 \% \text { loss in unfiltered } \mathrm{WW}\left(\mathrm{pH} 7.5,20-23^{\circ} \mathrm{C}\right) \text { and and } \\
\text { surface water }\left(\mathrm{pH} 6,20^{\circ} \mathrm{C}\right) \text {; Low: with activated sludge }>80 \% \\
\text { transformation over } 24 \mathrm{~h} \text { at } 21^{\circ} \mathrm{C}, \mathrm{pH} 7.4\end{array}$ & $\begin{array}{l}\text { Bisceglia and Lippa, 2014; Plósz et al., 2013; van } \\
\text { Nuijs et al., } 2012\end{array}$ \\
\hline & & & 3 & $\begin{array}{c}\text { Low: at neutral } \mathrm{pH} \text { at } 4^{\circ} \mathrm{C}, 9^{\circ} \mathrm{C}, 20^{\circ} \mathrm{C}, 23^{\circ} \mathrm{C} \& 31^{\circ} \mathrm{C} \text { over } 1-3 \mathrm{~d} \text {; after two } \\
\text { freeze-and-thaw cycles within } 3 \mathrm{~d}\end{array}$ & $\begin{array}{l}\text { Castiglioni et al., 2011a; Gheorghe et al., 2008; } \\
\text { González-Mariño et al., } 2010\end{array}$ \\
\hline & & nor- & 1 & $<5 \%$ change in unfiltered $\mathrm{WW}\left(\mathrm{pH} 7.4,19^{\circ} \mathrm{C}\right)$ & Baker and Kasprzyk-Hordern, 2011a \\
\hline
\end{tabular}




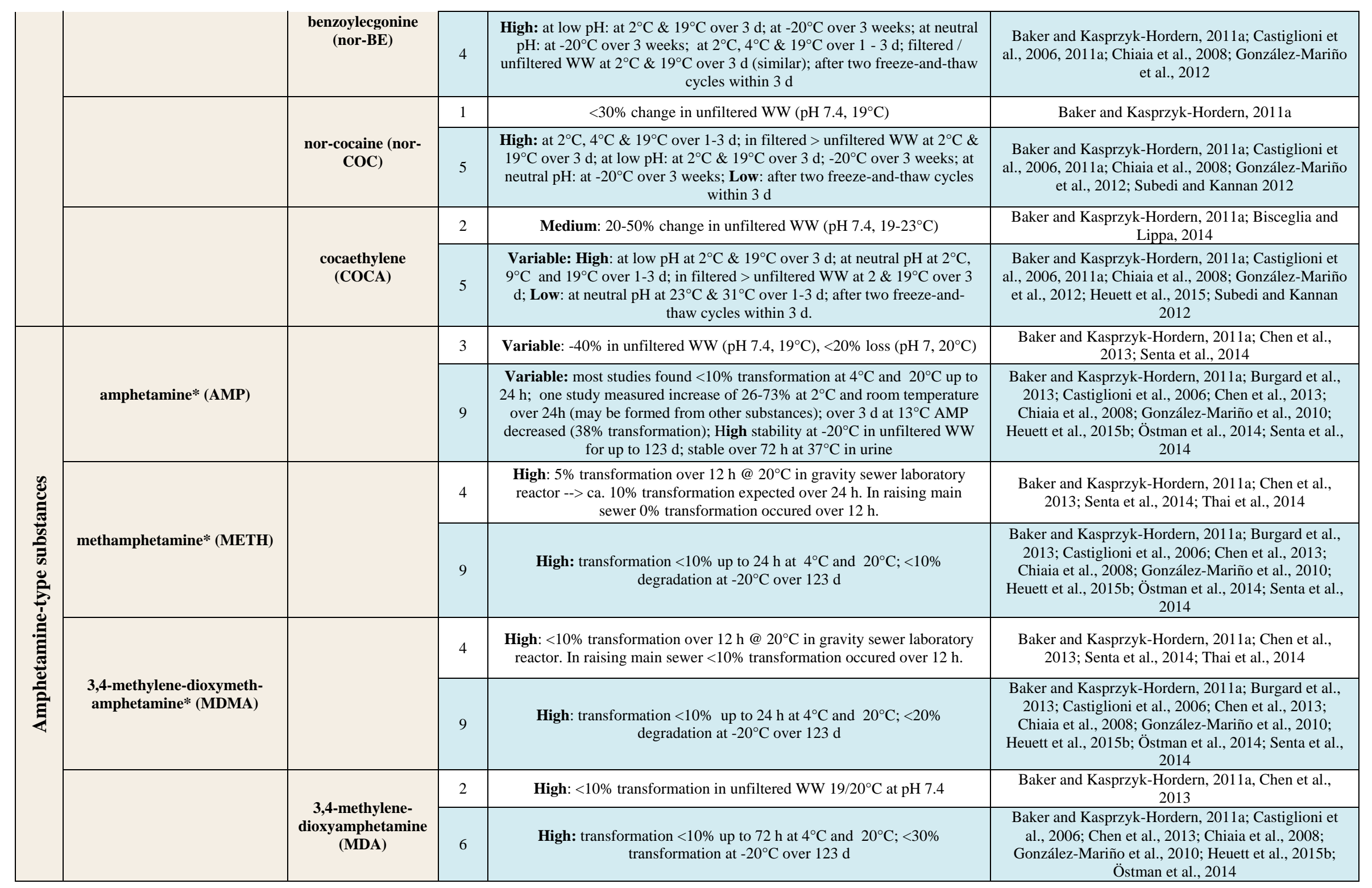




\begin{tabular}{|c|c|c|c|c|c|}
\hline & & \multirow{2}{*}{$\begin{array}{l}\text { 3,4-methylene- } \\
\text { dioxy-Nethyl- } \\
\text { amphetamine } \\
\text { (MDEA) }\end{array}$} & 1 & $<10 \%$ transformation in unfiltered $\mathrm{WW} 19^{\circ} \mathrm{C}$ at $\mathrm{pH} 7.4$ & Baker and Kasprzyk-Hordern, 2011a \\
\hline & & & 6 & $\begin{array}{l}\text { High: transformation }<10 \% \text { up to } 24 \mathrm{~h} \text { at } 4^{\circ} \mathrm{C} \text { and } 20^{\circ} \mathrm{C} ;<20 \% \\
\text { degradation at }-20^{\circ} \mathrm{C} \text { over } 123 \mathrm{~d}\end{array}$ & $\begin{array}{l}\text { Baker and Kasprzyk-Hordern, 2011a; Castiglioni et } \\
\text { al., 2006; Chiaia et al., 2008; González-Mariño et al., } \\
\text { 2010; Heuett et al., 2015b; Östman et al., } 2014\end{array}$ \\
\hline & \multirow{2}{*}{$\begin{array}{l}\text { methylbenzodioxolyl- } \\
\text { butanamine (MBDB) }\end{array}$} & & 1 & $<20 \%$ transformation in unfiltered $\mathrm{WW} 19^{\circ} \mathrm{C}$ at $\mathrm{pH} 7.4$ & Baker and Kasprzyk-Hordern, 2011a \\
\hline & & & 2 & High: transformation $<10 \%$ up to $24 \mathrm{~h}$ at $4^{\circ} \mathrm{C}$ and $20^{\circ} \mathrm{C}$ & $\begin{array}{c}\text { Baker and Kasprzyk-Hordern, 2011a; Östman et al., } \\
2014\end{array}$ \\
\hline & \multirow{2}{*}{$\begin{array}{l}\text { methylenedioxypyrovalerone* } \\
\text { (MDPV) }\end{array}$} & & 1 & in unfiltered WW no transformation at $22^{\circ} \mathrm{C}$ & Mardal et al. 2014 \\
\hline & & & 1 & $\begin{array}{l}\text { transformation }<10 \% \text { after } 24 \mathrm{~h} \text { at } 22^{\circ} \mathrm{C} \text { in wastewater; in urine stable over } \\
\qquad 14 \mathrm{~d} \text { at room temperature, } 4^{\circ} \mathrm{C} \text { and }-20^{\circ} \mathrm{C}\end{array}$ & Mardal et al. 2014 \\
\hline & \multirow{4}{*}{ methylphenidate* (ritalin) } & & 0 & NA & NA \\
\hline & & & 1 & $\begin{array}{l}36 \% \text { transformation at } 4{ }^{\circ} \mathrm{C} \text { and } 88 \% \text { transformation at room temperature } \\
\text { over } 24 \mathrm{~h} \text { in wastewater; in milliQ }<10 \% \text { transformation }\end{array}$ & Östman et al., 2014 \\
\hline & & \multirow{2}{*}{ ritalinic acid* } & 0 & NA & NA \\
\hline & & & 1 & $<10 \%$ transformation after $72 \mathrm{~h}$ in wastewater at $20^{\circ} \mathrm{C}$ & Burgard et al., 2013 \\
\hline & \multirow[b]{2}{*}{ mephedrone* } & & 0 & NA & NA \\
\hline & & & 1 & $\begin{array}{l}\text { in wastewater }<10 \% \text { transformation during } 24 \mathrm{~h} \text { at room temperature and at } \\
\qquad 4^{\circ} \mathrm{C}\end{array}$ & Östman et al., 2014 \\
\hline \multirow{7}{*}{$\frac{\mathscr{U}}{\tilde{\sigma}}$} & \multirow{4}{*}{ heroin } & & 1 & in unfiltered $\mathrm{WW}>90 \%$ transformation at $19^{\circ} \mathrm{C}$ and $\mathrm{pH} 7.4$ & Baker and Kasprzyk-Hordern, 2011a \\
\hline & & & 3 & $\begin{array}{l}\text { Low: transformed } 66 \% \text { after } 12 \mathrm{~h} \text { at } 2{ }^{\circ} \mathrm{C} \text { and } 79 \% \text { at } 19^{\circ} \mathrm{C} ; 50 \% \\
\text { degradation at }-20^{\circ} \mathrm{C} \text { over } 7 \mathrm{~d} \text { and }>90 \% \text { over } 123 \mathrm{~d}\end{array}$ & $\begin{array}{l}\text { Baker and Kasprzyk-Hordern, 2011a; González- } \\
\text { Mariño et al., 2010; Heuett et al., 2015b }\end{array}$ \\
\hline & & \multirow{2}{*}{$\begin{array}{l}\text { 6-mono-acetyl- } \\
\text { morphine* (6- } \\
\text { MAM) }\end{array}$} & 3 & $\begin{array}{l}\text { Low: } 88 \% \text { transformation over } 12 \mathrm{~h} @ 20^{\circ} \mathrm{C} \text { in gravity sewer laboratory } \\
\text { reactor --> ca. } 100 \% \text { transformation expected over } 24 \mathrm{~h} \text {. In raising main } \\
\text { sewer } 87 \% \text { transformation occured over } 12 \mathrm{~h} \text {. }\end{array}$ & $\begin{array}{l}\text { Baker and Kasprzyk-Hordern, 2011a; Senta et al., } \\
\text { 2014; Thai et al., } 2014\end{array}$ \\
\hline & & & 5 & $\begin{array}{l}\left.\text { Low: degraded quickly in WW ( } 6 \% \text { transformation at } 20^{\circ} \mathrm{C} \text { over } 12 \mathrm{~h}\right) \text {; but } \\
\text { relatively stable in milliQ; High: }<20 \% \text { degradation at }-20^{\circ} \mathrm{C} \text { over } 3,7,17 \text {, } \\
27 \mathrm{~d}\end{array}$ & $\begin{array}{l}\text { Baker and Kasprzyk-Hordern, 2011a; Castiglioni et } \\
\text { al., 2006; Heuett et al., 2015b; Östman et al., 2014; } \\
\text { Senta et al., } 2014\end{array}$ \\
\hline & \multirow{3}{*}{ methadone* (MTD) } & & 3 & $\begin{array}{l}\text { Variable: in unfiltered WW }<10 \% \text { loss at } 19 / 20^{\circ} \mathrm{C} \text { and } \mathrm{pH} 7.4 ;+10 \% \text { at } \\
20^{\circ} \mathrm{C} \text { in unfiltered } \mathrm{WW} \mathrm{pH} 7.5 ; \text { may be prone to sorption }\end{array}$ & $\begin{array}{l}\text { Baker and Kasprzyk-Hordern, 2011a; Senta et al., } \\
\text { 2014; van Nuijs et al., } 2012\end{array}$ \\
\hline & & & 8 & $\begin{aligned} \text { Variable: } & <20 \% \text { difference at room temperature and } 4^{\circ} \mathrm{C} \text {; may be prone to } \\
& \text { sorption; }>40 \% \text { degradation at }-20^{\circ} \mathrm{C} \text { over } 123 \mathrm{~d}\end{aligned}$ & $\begin{array}{l}\text { Baker and Kasprzyk-Hordern, 2011a; Castiglioni et } \\
\text { al., 2006; Rosa Boleda et al., 2011; Chiaia et al., 2008; } \\
\text { González-Mariño et al., 2010; Heuett et al., 2015b; } \\
\text { Östman et al., 2014; Senta et al., } 2014\end{array}$ \\
\hline & & $\begin{array}{l}\text { 2-ethylidene-1,5- } \\
\text { dimethyl-3,3- }\end{array}$ & 1 & $\begin{array}{c}\text { in unfiltered WW ca. } 20 \% \text { loss at } 19^{\circ} \mathrm{C} \text { and } \mathrm{pH} 7.4 \text {; may be prone to } \\
\text { sorption }\end{array}$ & Baker and Kasprzyk-Hordern, 2011a \\
\hline
\end{tabular}

\section{Page $\mathbf{1 6}$ of $\mathbf{3 8}$}




\begin{tabular}{|c|c|c|c|c|c|}
\hline & & $\begin{array}{l}\text { diphenyl- } \\
\text { pyrrolidine* } \\
\text { (EDDP) }\end{array}$ & 5 & $\begin{array}{l}\text { Variable: less than } 15 \% \text { difference after } 24 \mathrm{~h} \text {; may be prone to sorption; ca. } \\
\qquad 40 \% \text { degradation at }-20^{\circ} \mathrm{C} \text { over } 3,7 \text { and } 123 \mathrm{~d}\end{array}$ & $\begin{array}{l}\text { Baker and Kasprzyk-Hordern, 2011a; Rosa Boleda et } \\
\text { al., 2011; Castiglioni et al., 2006; Heuett et al., 2015b; } \\
\text { Östman et al., 2014 }\end{array}$ \\
\hline & \multirow{4}{*}{ morphine* (MOR) } & \multirow[b]{4}{*}{ 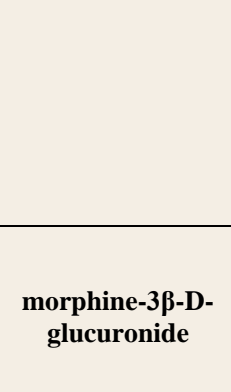 } & 2 & $\begin{array}{l}\text { Variable: in unfiltered WW up to } 50 \% \text { loss }\left(19^{\circ} \mathrm{C}, \mathrm{pH} 7.4\right) \text {; up to } 20 \% \\
\text { increase }\left(20^{\circ} \mathrm{C}, \mathrm{pH} 7.5\right) \text { (transformation product of other substances) }\end{array}$ & $\begin{array}{l}\text { Baker and Kasprzyk-Hordern, 2011a; Senta et al., } \\
2014\end{array}$ \\
\hline & & & 5 & $\begin{array}{l}\text { Variable: difficult to judge because of the generation of morphine from } \\
\text { other drugs; generally relativ high stablity at } 4^{\circ} \mathrm{C} \text { in unfiltered WW over } 24 \\
\text { h; }<20 \% \text { degradation at }-20^{\circ} \mathrm{C} \text { over } 3,7,17,27 \mathrm{~d}\end{array}$ & $\begin{array}{l}\text { Baker and Kasprzyk-Hordern, 2011a; Castiglioni et } \\
\text { al., 2006; Heuett et al., 2015b; Östman et al., 2014; } \\
\text { Senta et al., } 2014\end{array}$ \\
\hline & & & 1 & complete transformation in unfiltered $\mathrm{WW} \mathrm{pH} 7.520^{\circ} \mathrm{C}$ & Senta et al., 2014 \\
\hline & & & 2 & $\begin{array}{c}\text { Low: at } \mathrm{pH} 7.5 \text { over } 24 \mathrm{~h} \text { at } 20^{\circ} \mathrm{C}>80 \% \text { transformation in } \mathrm{WW} ; 96 \% \\
\text { transformation in WW at } 4^{\circ} \mathrm{C} \text { over } 3 \mathrm{~d} \text {; high: in WW at } \mathrm{pH} 2 \text { over } 78 \mathrm{~h} \text { at } \\
220^{\circ} \mathrm{C}\end{array}$ & Castiglioni et al., 2006; Senta et al., 2014 \\
\hline & \multirow[b]{2}{*}{ oxycodone* } & & 1 & $<10 \%$ transformation in unfiltered $\mathrm{WW}$ at $19^{\circ} \mathrm{C}$ and $\mathrm{pH} 7.4$ & Baker and Kasprzyk-Hordern, 2011a \\
\hline & & & 4 & $\begin{array}{l}\text { High: stable }\left(<20 \% \text { transformation) at } 19^{\circ} \mathrm{C} \text { and } \mathrm{pH} 7.4 ;<10 \% \text { degradation }\right. \\
\text { at }-20^{\circ} \mathrm{C} \text { over } 3,7,17,27,123 \mathrm{~d}\end{array}$ & $\begin{array}{l}\text { Baker and Kasprzyk-Hordern, 2011a; Castiglioni et } \\
\text { al., 2006; Heuett et al., 2015b; Östman et al., } 2014\end{array}$ \\
\hline & \multirow[b]{2}{*}{ fentanyl* } & & 1 & $\begin{array}{c}<20 \% \text { transformation in unfiltered } \mathrm{WW} \text { at } 19^{\circ} \mathrm{C} \text { and } \mathrm{pH} 7.4 ; \text { may be prone } \\
\text { to sorption }\end{array}$ & Baker and Kasprzyk-Hordern, 2011a \\
\hline & & & 3 & $\begin{array}{l}\text { Variable/medium: }<10 \% \text { degradation in filtered } \mathrm{WW} \text { at room temperature; } \\
<20 \% \text { loss in unfiltered } \mathrm{WW} 19^{\circ} \mathrm{C} \text { and } \mathrm{pH} 7.4 \text { but } 62 \% \text { loss after } 72 \mathrm{~h} \text { under } \\
\text { same conditions; may be prone to sorption high: in milliQ }\end{array}$ & $\begin{array}{l}\text { Baker and Kasprzyk-Hordern, 2011a; Rosa Boleda et } \\
\text { al., 2011; Östman et al., } 2014\end{array}$ \\
\hline & \multirow[b]{2}{*}{ buprenorphine* } & & 1 & $<10 \%$ transformation in unfiltered $\mathrm{WW}$ at $19^{\circ} \mathrm{C}$ and $\mathrm{pH} 7.4$ & Baker and Kasprzyk-Hordern, 2011a \\
\hline & & & 1 & $\begin{array}{l}\text { Variable: ca.30\% formation in filtered } \mathrm{WW} \text { at room temperature; }<10 \% \\
\text { degradation in unfiltered } \mathrm{WW} \text { at } 4{ }^{\circ} \mathrm{C} \text { and } 19^{\circ} \mathrm{C} \text { and } \mathrm{pH} 7.4 ; \text { high: in milliQ } \\
<10 \% \text { transformation }\end{array}$ & $\begin{array}{l}\text { Baker and Kasprzyk-Hordern, 2011a; Östman et al., } \\
\qquad 2014\end{array}$ \\
\hline & \multirow[b]{2}{*}{ codeine* (COD) } & & 1 & High: $<20 \%$ transformation/formed in unfiltered $\mathrm{WW}$ at $19^{\circ} \mathrm{C}$ and $\mathrm{pH} 7.4$ & Baker and Kasprzyk-Hordern, 2011a \\
\hline & & & 4 & $\begin{array}{l}\text { Variable: High: In filtered/unfiltered WW }<20 \% \text { transformation over } 24 \mathrm{~h} \\
\text { at } 4^{\circ} \mathrm{C}, 19^{\circ} \mathrm{C} \text { and room temperature; may be formed from other substances; } \\
<10 \% \text { degradation at }-20^{\circ} \mathrm{C} \text { over } 3,7,17,27,123 \mathrm{~d} ; \text { Low: } 80 \% \text { transformed } \\
\text { in diluted activated sludge } \mathrm{pH} 7\end{array}$ & $\begin{array}{l}\text { Baker and Kasprzyk-Hordern, 2011a; Heuett et al., } \\
\text { 2015b; Östman et al., 2014; Wick et al., } 2011\end{array}$ \\
\hline \multirow{3}{*}{  } & \multirow[b]{2}{*}{ tetrahydro-cannabinol (THC) } & & 1 & $\begin{array}{c}<20 \% \text { transformation in unfiltered } \mathrm{WW} \mathrm{pH} 7.520^{\circ} \mathrm{C} \text {; may be lost due to } \\
\text { sorption }\end{array}$ & Senta et al., 2014 \\
\hline & & & 1 & $\begin{array}{l}\text { NA - almost no THC is excreted in human urine (Karch and Jenkins, 2006; } \\
\text { Postigo et al., 2011; Lai et al., 2011); in spiked unfiltered WW stored at - } \\
20^{\circ} \mathrm{C} 50 \% \text { degradation over } 7 \text { days and }>90 \% \text { after } 123 \mathrm{~d}\end{array}$ & Heuett et al., 2015b \\
\hline & & ТHC-COOH* & 1 & $\begin{array}{c}<20 \% \text { transformation in unfiltered } \mathrm{WW} \text { pH } 7.520^{\circ} \mathrm{C} \text {; may be lost due to } \\
\text { sorption }\end{array}$ & Senta et al., 2014 \\
\hline
\end{tabular}




\begin{tabular}{|c|c|c|c|c|c|}
\hline & & & 4 & $\begin{array}{l}\text { Variable: may be lost due to sorption; High in } \mathrm{WW} \text { at } 4^{\circ} \mathrm{C} \text { and } 20^{\circ} \mathrm{C} \text { over } \\
72 \mathrm{~h} \text {; high on SPE cartridges over three weeks at }-20^{\circ} \mathrm{C} \text {; high at }-20^{\circ} \mathrm{C} \text { over } \\
3,7,17,27,123 \mathrm{~d} \text {; Low at } \mathrm{pH} 2 \text { over } 24 \mathrm{~h}\end{array}$ & $\begin{array}{l}\text { Boix et al., 2014; Castiglioni et al., 2006; González- } \\
\text { Mariño et al., 2010; Heuett et al., 2015b; Senta et al. } \\
\qquad 2014\end{array}$ \\
\hline & & & 1 & $<20 \%$ transformation in unfiltered $\mathrm{WW}$ pH $7.520^{\circ} \mathrm{C}$ & Senta et al., 2014 \\
\hline & & & 1 & $<20 \%$ transformation at $\mathrm{pH} 7.4, \mathrm{pH} 2,10^{\circ} \mathrm{C}$ and $20^{\circ} \mathrm{C}$ in unfiltered $\mathrm{WW}$ & Senta et al., 2014 \\
\hline \multirow{8}{*}{ 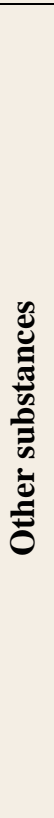 } & \multirow[b]{2}{*}{ ketamine* (KET) } & & 2 & High: $<10 \%$ transformation in unfiltered $\mathrm{WW} \mathrm{pH} 7.5,20^{\circ} \mathrm{C}$ & $\begin{array}{l}\text { Baker and Kasprzyk-Hordern, 2011a; Castiglioni et } \\
\text { al., } 2015\end{array}$ \\
\hline & & & 3 & $\begin{array}{c}\text { High: at } 4^{\circ} \mathrm{C} \text { and room temperature at } \mathrm{WW} \mathrm{pH} \text { and acidified to } \mathrm{pH} 4 \text { and in } \\
\text { milliQ water at } 4^{\circ} \mathrm{C} \text { and room temperature }\end{array}$ & $\begin{array}{l}\text { Baker and Kasprzyk-Hordern, 2011a; Castiglioni et } \\
\text { al., 2015; Östman et al., } 2014\end{array}$ \\
\hline & & \multirow{2}{*}{$\begin{array}{l}\text { norketamine } \\
(\text { norKET) }\end{array}$} & 2 & High: $<10 \%$ transformation in unfiltered $\mathrm{WW} \mathrm{pH} 7.5,20^{\circ} \mathrm{C}$ & $\begin{array}{l}\text { Baker and Kasprzyk-Hordern, 2011a; Castiglioni et } \\
\text { al., } 2015\end{array}$ \\
\hline & & & 3 & $\begin{array}{c}\text { High: at } 4^{\circ} \mathrm{C} \text { and room temperature at } \mathrm{WW} \mathrm{pH} \text { and acidified to } \mathrm{pH} 4 \text { and in } \\
\text { milliQ water at } 4^{\circ} \mathrm{C} \text { and room temperature }\end{array}$ & $\begin{array}{l}\text { Baker and Kasprzyk-Hordern, 2011a; Castiglioni et } \\
\text { al., 2015; Östman et al., } 2014\end{array}$ \\
\hline & \multirow[b]{2}{*}{$\begin{array}{l}\text { lysergic acid diethylamide* } \\
\text { (LSD) }\end{array}$} & & 1 & $<10 \%$ transformation in unfiltered $\mathrm{WW} \mathrm{pH} 7.5,20^{\circ} \mathrm{C}$ & Baker and Kasprzyk-Hordern, 2011a \\
\hline & & & 4 & $\begin{array}{l}\text { High: in WW pH and acidified to } \mathrm{pH} 2 \text { at room temperature and at } 4^{\circ} \mathrm{C} \text {; } \\
\text { Medium: in WW at room temperature up to } 24 \% \text { transformation; in milliQ } \\
\text { water at } 44^{\circ} \mathrm{C} \text { and room temperature ( } 40 \% \text { transformation); over } 3,7,17,27 \text {, } \\
\qquad 123 \mathrm{~d} \text { at }-20^{\circ} \mathrm{C}>20 \text { and }<50 \% \text { degradation }\end{array}$ & $\begin{array}{l}\text { Baker and Kasprzyk-Hordern, 2011a; Chiaia et al., } \\
\text { 2008; Heuett et al., 2015b; Östman et al., } 2014\end{array}$ \\
\hline & & \multirow[b]{2}{*}{$\begin{array}{l}\text { 2-oxo-3-hydroxy- } \\
\text { LSD }\end{array}$} & 1 & $<20 \%$ transformation in unfiltered $\mathrm{WW} \mathrm{pH} 7.5,20^{\circ} \mathrm{C}$ & Baker and Kasprzyk-Hordern, 2011a \\
\hline & & & 3 & $\begin{array}{l}\text { High: at } \mathrm{WW} \mathrm{pH} \text { and acidified to } \mathrm{pH} 2 \text { at room temperature and at } 4^{\circ} \mathrm{C} \text {; in } \\
\text { milliQ water at } 4^{\circ} \mathrm{C} \text { and room temperature }\end{array}$ & $\begin{array}{l}\text { Baker and Kasprzyk-Hordern, 2011a; Chiaia et al., } \\
\text { 2008; Östman et al., } 2014\end{array}$ \\
\hline
\end{tabular}




\subsection{Cocaine and metabolites}

In-sample stability of cocaine (COC) and its metabolites has been widely studied over a range of different conditions. These studies focused mostly on in-sample stability of COC and its main metabolite, benzoylecgonine (BE), which is used for back-calculation. Stability of COC was generally low under all tested conditions (Table 2). Hydrolysis of COC seems pH-dependent (Warner and Norman, 2000), and acidification of the sample can preserve COC concentrations at low and high temperatures over at least three days (Table 2). Transformation of COC can also be prevented using preservatives (e.g., sodium metabisulfite $\left(\mathrm{Na}_{2} \mathrm{~S}_{2} \mathrm{O}_{5}\right)$, sodium azide $\left(\mathrm{NaN}_{3}\right)$ ), and COC concentration changes seemed negligible after extraction onto SPE cartridges (HLB) (Chen et al., 2013; GonzálezMariño et al., 2010).

The wastewater matrix seems to have an influence on the extent of transformation (Castiglioni et al., 2006, 2011a; Gheorghe et al., 2008). COC concentrations remained slightly higher over three days in unfiltered samples compared to filtered wastewater $\left(20^{\circ} \mathrm{C}, \mathrm{pH}\right.$ 7) (Chen et al., 2013). At $-20^{\circ} \mathrm{C}$ and $\mathrm{pH}$ 7, COC concentrations in unfiltered wastewater only slightly decreased over three weeks (Chiaia et al., 2008), and another study reported that COC can be stable over $24 \mathrm{~h}$ in Milli-Q water at $4^{\circ} \mathrm{C}$ and $25^{\circ} \mathrm{C}$ (Östman et al., 2014). Furthermore, it is important to note that sorption to SPM of COC and its metabolites seems to be negligible (Baker and Kasprzyk-Hordern, 2011b), but COC concentrations in wastewater samples decreased during freeze-and-thaw cycles (Castiglioni et al., 2011).

In-sewer stability of COC was relatively low over $12 \mathrm{~h}$ at $\mathrm{pH} 7.1-7.5$ and $20^{\circ} \mathrm{C}$ in a study considering aerobic and anaerobic sewer biofilms, in which transformation of $\mathrm{COC}$ appeared to be stronger under aerobic conditions, compared to anaerobic in-sewer conditions (Thai et al., 2014).

Unlike COC, its main metabolite BE showed high in-sample stability under various conditions (Table 2). In-sewer stability of $\mathrm{BE}$ with aerobic and anaerobic biofilms has been shown to be high at $20^{\circ} \mathrm{C}$ over 12h (Thai et al., 2014), whereas one study revealed a decrease of BE under aerobic and anaerobic conditions with activated sludge biomass at $21^{\circ} \mathrm{C}$ over $24 \mathrm{~h}$ (Plósz et al., 2013). Information on other COC metabolites is listed in Table 2. 


\subsection{Amphetamine and amphetamine-type substances}

This group encompasses compounds with chemical structures similar to that of amphetamine (AMP). It includes methamphetamine (METH), 3,4-methylenedioxymethamphetamine (MDMA) and its metabolite 3,4-methylenedioxyamphetamine (MDA), 3,4-methylenedioxy-N-ethylamphetamine (MDEA), methylbenzodioxolylbutanamine (MBDB), as well as, the novel synthetic cathinones, cathinones $\mathrm{HCl}$, methylenedioxypyrovalerone (MDPV), mephedrone, methylphenidate (ritalin) and its metabolite, ritalinic acid.

In-sample stability of AMP in unfiltered wastewater has been consistently shown to be high at $\mathrm{pH} 7$ at $4^{\circ} \mathrm{C}$ and $20^{\circ} \mathrm{C}$ for $24 \mathrm{~h}$ in most studies (Table 2). However, Baker et al. 2011a reported an increase (26\%) of AMP concentrations at $2^{\circ} \mathrm{C}$ and $\mathrm{pH} 7$ and a $73 \%$ increase in AMP at room temperature over 24h. AMP is also a metabolite of METH, and the pharmaceuticals, selegiline and dextroamphetamine (Kraemer and Maurer, 2002; Heuett et al., 2015a). However, in-sample stability of METH is high in unfiltered wastewater at $\mathrm{pH} 7$ at $4{ }^{\circ} \mathrm{C}$ and room temperature (Table 2) and substantial in-sewer transformation of METH to AMP is thus unlikely. AMP and METH were reported as highly stable under all tested conditions, particularly after addition of $\mathrm{NaN}_{3}$ to the samples over three weeks, instant freezing $\left(-20^{\circ} \mathrm{C}\right)$ over 123 days, and after acidification over three weeks (Table 2).

MDMA, MDA and MDEA showed high in-sample stability in unfiltered wastewater at $\mathrm{pH} 7$ and at $4^{\circ} \mathrm{C}$ and $20^{\circ} \mathrm{C}$ for $24 \mathrm{~h}$ (Table 2). In addition, they were stable after instant freezing $\left(-20^{\circ} \mathrm{C}\right)$ and acidification for $24 \mathrm{~h}$ up to three weeks. One study investigating the stability of MBDB in unfiltered wastewater at $2^{\circ} \mathrm{C}$ and at $20^{\circ} \mathrm{C}$, both at $\mathrm{pH} 7$, reported a medium stability with lower concentration after 12-24h (Baker and Kasprzyk-Hordern, 2011a).

The transformation of MDPV and 12 metabolites - three of them previously reported as human metabolites - was investigated in wastewater at $22^{\circ} \mathrm{C}$, and no significant decrease of MDPV (high insewer and in-sample stability) was observed in a 10-day experiment (Mardal and Meyer, 2014). Further experiments demonstrated the glucuronidase activity in wastewater, since the signal of four glucuronide phase II metabolites decreased by more than $99 \%$ after one day (Mardal and Meyer, 2014). 
One study in unfiltered wastewater found that in-sample stability of mephedrone is high at $4^{\circ} \mathrm{C}$ and room temperature over $24 \mathrm{~h}$ (Östman et al., 2014). Similarly, mephedrone was stable in urine at $4^{\circ} \mathrm{C}$, room temperature and $-20^{\circ} \mathrm{C}$ over at least 2 days (Johnson and Botch-Jones, 2013). Methylphenidate's in-sample stability in wastewater ranged from medium (after $24 \mathrm{~h}$ at $4^{\circ} \mathrm{C}$ ) to $l o w$ (at room temperature, Burgard et al., 2013; Östman et al., 2014). In Milli-Q water, methylphenidate was stable for 24h both at $4^{\circ} \mathrm{C}$ and at room temperature (Östman et al., 2014). Ritalinic acid had a high in-sample stability in wastewater for $72 \mathrm{~h}$ at $20^{\circ} \mathrm{C}$ (Burgard et al., 2013).

Few studies have investigated the influence of sewer biofilm or SPM on stability. The in-sewer stability study conducted by Thai et al. (2014) found a non-significant increase of METH (<5\% after 12h) in the presence of aerobic biofilm. Subedi et al. (2014) showed no sorption for METH, MDMA and MDEA and a medium loss $(30-40 \%)$ due to sorption to SPM for MDA and AMP. In contrast, Baker et al. (2011b) found <10\% of AMP sorbed to SPM (Baker and Kasprzyk-Hordern, 2011b).

In general, the in-sample stability of AMP, METH, MDMA, MDA and MDEA in unfiltered and filtered wastewater samples at different temperatures have yielded similar results, demonstrating that these compounds had a high stability with the exception of AMP, for which a higher change in concentration was reported in some experiments (>20\%) (Baker and Kasprzyk-Hordern, 2011a; Östman et al., 2014) (Table 2).

\subsection{Opiates}

Heroin use had been estimated by measuring its metabolite 6-monoacetyl morphine (6-MAM), however, 6-MAM itself has low in-sample stability and can transform quickly to morphine (MOR) in the wastewater matrix. A wastewater sample can lose up to $42 \%$ 6-MAM after $24 \mathrm{~h}$ at $19^{\circ} \mathrm{C}$ (Baker and Kasprzyk-Hordern, 2011a). Similarly, in-sewer stability is low, since up to 90\% of 6-MAM was lost at $20^{\circ} \mathrm{C}$ after $12 \mathrm{~h}$ in the study with sewer biofilms, performed by Thai et al. (2014). A reliable unbiased biomarker for heroin has not yet been found.

Although the stability of morphine (MOR) is high in wastewater samples, the estimation of use of heroin from MOR concentrations is difficult, because it is used itself as a pharmaceutical and is a metabolite of other opiates (e.g., ethyl morphine, 6-MAM, codeine (COD)). The associated 


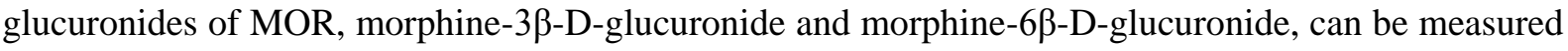
in wastewater, but they have a low stability and quickly deconjugate to MOR (Table 2).

Most of a COD dose is excreted with urine, either as unchanged COD or as a conjugate. COD is highly stable in wastewater samples (Table 2), and its consumption can, therefore, be estimated by measuring the load of COD in wastewater. Jelic et al. (2015) also reported high in-sewer stability. However, COD exhibited low stability in batch experiments with diluted activated sludge from wastewater treatment plants (Wick et al., 2011).

Most wastewater studies to date measured both methadone (MTD) and its main metabolite EDDP, but the consumption of MTD was only estimated using the parent compound. Both MTD and EDDP seem to be stable in wastewater both under in-sample storage conditions (at $4^{\circ} \mathrm{C}$, Östman et al., 2014) and simulated in-sewer conditions (at $19^{\circ} \mathrm{C}$ and $\mathrm{pH} 7$, van Nuijs et al., 2012). However, a portion of MTD and EDDP can adsorb to SPM in wastewater. Baker and Kasprzyk-Hordern (2011a) observed a significant reduction of spiked MTD (23\%) and EDDP (72\%) in unfiltered wastewater after $72 \mathrm{~h}$ at $19^{\circ} \mathrm{C}$, most likely due to sorption processes. Overall, the stability of MTD and EDDP is variable depending on the conditions and SPM/biofilm content.

Buprenorphine has a variable stability in wastewater samples (Table 2). It is a relatively hydrophobic compound and eliminated primarily via feces as free drug with low concentrations occurring in urine. Oxycodone and fentanyl are therapeutic opiates that receive increasing attention as drugs of abuse. To date, all wastewater studies used the parent compounds as biomarkers, although each drug has specific metabolites (e.g., noroxycodone and norfentanyl, Baselt, 2008). Östman et al. (2014) found both compounds stable under storage condition, but Baker and Kasprzyk-Hordern (2011) observed significant degradation of fentanyl in unfiltered wastewater $(62 \%)$ after $72 \mathrm{~h}$ at $19^{\circ} \mathrm{C}$ and $\mathrm{pH}$ 7.4. This again may be attributed to adsorption to SPM, since only $6 \%$ of fentanyl was lost during the same period in filtered wastewater. Based on the reviewed studies, fentanyl had a medium in-sample stability, whereas oxycodone was highly stable. 


\subsection{Cannabinoids}

Cannabis's primary active compound is $\Delta 9$-tetrahydrocannabinol (THC), which after consumption is metabolized to more than 20 metabolites, the two main ones being 11-nor-9-carboxy-THC (THC$\mathrm{COOH}$ ) and 11-hydroxy-THC (THC-OH) (Karch and Jenkins, 2006). These metabolites are excreted as glucuronide conjugates, however in wastewater, they are hydrolyzed/deconjugated to the parent metabolite (Castiglioni et al., 2008). For this reason, THC-COOH is normally used to estimate cannabis consumption in WBE studies (Castiglioni et al., 2011b; Lai et al., 2011). However, there are analytical difficulties especially associated with the sample treatment and detection of THC-COOH due to its higher lipophilicity compared to other illicit drugs (Vazquez-Roig et al., 2013). This sometimes may hamper the inclusion of $\mathrm{THC}-\mathrm{COOH}$ in analytical methods for routine multi-class determination of illicit drugs.

In-sample stability tests with raw wastewater ( $\mathrm{pH} 7-8$ ) showed high stability over $72 \mathrm{~h}$ with minimal impact of temperature (Table 2). However, after longer storage times at $4^{\circ} \mathrm{C}$, degradation became more significant after seven days (González-Mariño et al., 2010). Frozen samples were stable up to 4 months (Heuett et al., 2015b). Acidification of samples to $\mathrm{pH} 2$ (with $\mathrm{H}_{3} \mathrm{PO}_{4}$ ) increased the transformation of THC-COOH and THC-OH in wastewater (Khan and Nicell, 2012; Senta et al., 2014). At pH 2, THC-COOH was found to have enhanced adsorption (loss of 54\%), compared to only $10 \%$ loss at unadjusted pH 7.4 (Senta et al., 2013). This was also found in a similar study where only $1.3 \%$ of THC-COOH was expected to have adsorbed to SPM at environmental $\mathrm{pH}$ conditions $(\mathrm{pH} \approx$ 7.5) (Khan and Nicell, 2012).

\subsection{Other substances}

The stability of lysergic acid diethylamide (LSD) in wastewater at $\mathrm{pH} 7.4$ and acidified to $\mathrm{pH} 2$ at room temperature and at $4^{\circ} \mathrm{C}$ after $24 \mathrm{~h}$ was high (Table 2). The metabolite 2-oxo-3-hydroxy-LSD also exhibits high stability and only slightly decreased (10-20\%) in wastewater $\mathrm{pH} 7.4$ at room temperature after $24 \mathrm{~h}$, while acidification and/or lowering the temperature prevented this (Baker and KasprzykHordern, 2011a; Chiaia et al., 2008). 
The in-sample stability of ketamine (KET) and its metabolite norKET was studied for different conditions: temperature $\left(4^{\circ} \mathrm{C}\right.$, room temperature), $\mathrm{pH}(2,7.4)$, and time (12-72h) (Table 2). Both KET and norKET had a high stability in all analyzed conditions.

\section{Discussion}

The reviewed studies clearly show that concentrations of several substances decreased in unfiltered wastewater under different conditions. In order to explain discrepancies for a given biomarker among different studies with variable wastewater matrices, the effects of chemical, biological and physical processes need to be considered.

\section{Biological and chemical transformation processes}

In the absence of appropriate abiotic controls, it is difficult to differentiate between chemical and biological transformations. Several of the investigated biomarkers, e.g., COC and 6-MAM, have chemical structures (esters) that are prone to abiotic or biotic hydrolysis in wastewater. Abiotic control experiments were only carried out in three studies (Wick et al., 2011; Mardal and Meyer, 2014; Senta et al., 2014).

Other important, chemically or biologically mediated, processes are conjugation and deconjugation.

THC, morphine and MDPV are excreted in conjugated form (e.g., as glucuronides and sulfates) and tend to deconjugate during in-sewer transport (Boleda et al., 2007; D'Ascenzo et al., 2003; Evgenidou, 2015, Mardal and Meyer, 2014).

Studies of biological transformation (biotransformation) mechanisms related to biomarkers in sewers are scarce (Mardal and Meyer, 2014). Biotransformation can occur under aerobic and anaerobic conditions, whereby illicit drugs demonstrate affinity for bacterial enzymes and serve as co-metabolic (non-growth) substrates (Siegrist and Joss, 2012). In most of the reviewed stability studies, the redox conditions (aerobic/anaerobic) were not measured or monitored, even though the redox potential influences bacterial activity, and biotransformation is higher under aerobic than anaerobic conditions (Thai et al., 2014). The extent of biotransformation, therefore, depends on the type and amount of active biomass in the sewer, which may vary among different networks (Roth and Lemmer, 1994). 
It is still not well understood which species of bacteria are responsible for biological transformation of organic micropollutants in wastewater treatment processes (Siegrist and Joss, 2012). Further, the microbial community in wastewater treatment plants deviates from sewer communities (HvitvedJacobsen et al., 2013). The conditions in the activated sludge process in wastewater treatment are selected to favor growth of specific microorganisms, such as nitrifying and phosphorous-accumulating bacteria (Henze et al., 2002). Under the conditions prevailing in sewers, fast-growing heterotrophic bacteria outcompete the slower growing organisms, such as nitrifying bacteria (Hvitved-Jacobsen et al., 2013). Therefore, the use of activated sludge to mimic in-sewer transformation may not be representative for the active microbial community in sewers. Only one study that investigated the stability of illicit drug biomarkers included sewer wall biofilm (Thai et al., 2014), and the results implied that biofilm is an important parameter that needs to be taken into account.

Another factor that may explain some of the variability in stability is the influence of other organic and inorganic constituents of SPM, such as feces and toilet paper (natural polymers, cellulose). These factors have not yet been investigated for their potential effect on transformations.

Two of the key environmental variables influencing chemical and biological reactions are temperature and $\mathrm{pH}$. Most studies reported or investigated the effect of these variables on the stability. Wastewater temperatures in sewers can vary from $10^{\circ} \mathrm{C}$ in winter up to $30^{\circ} \mathrm{C}$ in summer (Tchobanoglous and Burton, 1991). The reviewed transformation studies were conducted at constant temperature, either at low temperature $\left(2-10^{\circ} \mathrm{C}\right)$ or at room temperature $\left(19-23^{\circ} \mathrm{C}\right)$. Results show a temperature dependence

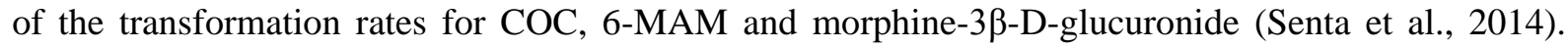
Similarly, (chemical and biological) hydrolysis rates of $\mathrm{COC}$ in wastewater were higher at $31^{\circ} \mathrm{C}$ compared to $23^{\circ} \mathrm{C}$ and $9{ }^{\circ} \mathrm{C}$. The transformation rate coefficients were larger than those reported in deionized water at similar $\mathrm{pH}$ and temperatures, confirming that biologically mediated hydrolysis can play an important role in wastewater (Bisceglia, 2007; Bisceglia and Lippa, 2014).

The reviewed studies typically state one $\mathrm{pH}$ value, omitting whether $\mathrm{pH}$ was recorded at the beginning or end of the experiment or frequently monitored. A recent study found that biotransformation is $\mathrm{pH}-$ dependent, where the neutral fraction of ionizable substances (e.g., amines) induced a higher microbial uptake (Gulde et al., 2014). During the transport of illicit drug biomarkers in sewers or over the course 
of an experiment, transformations of macronutrients in the wastewater can change the $\mathrm{pH}$ (Sharma et al., 2013), which may change the bioavailability of biomarkers with pKas close to the wastewater $\mathrm{pH}$.

\section{Spiking concentration levels}

All studies conducted multi-target analysis, which can make the interpretation of results difficult for some substances, if both parent compounds and metabolites were spiked together. To avoid this, one study spiked mass-labelled analogues to be able to differentiate between parent compound and metabolite transformations in separate batch experiments (Thai et al., 2014).

Another limitation may arise if the selected excreted human biomarker is also a transformation product formed in the sewer (Heuett et al. 2015a). For example, the stability of an excreted metabolite may be low, but as a result of in-sewer transformation from the parent compound, a stability study may nonetheless exhibit constant concentration levels over the investigated period (as in the case of

$415 \mathrm{COC} / \mathrm{BE})$. One approach to tackle this problem, then, is to use several biomarkers of one parentcompound to more reliably back-calculate the consumption (Heuett et al., 2015a; Baker et al., 2014). This method is, however, only feasible when multiple metabolites are available and stable in the sewer.

Spiking levels in the studies spanned from zero, i.e., relying on the concentrations already present in the wastewater, up to $100 \mu \mathrm{gL}^{-1}$. It is necessary to distinguish between pathway investigation studies, where high spikes seem appropriate, and studies to monitor biomarker stability at environmentally relevant concentrations. Testing guidelines (e.g., Organization for Economic Cooperation and Development (OECD)) recommend high concentrations mostly to reduce analytical constraints. However, at high concentrations, microorganisms may need time to adapt before starting to transform illicit drugs (Mardal and Meyer, 2014). Thai et al. (2014) found no impact of high spiking levels on the transformation of COC, BE, MDMA and 6-MAM in their in-sewer stability study. Whether high levels of other illicit drugs may even inhibit biotransformation was not investigated and may require further comparative studies.

Another, so far overlooked, aspect is the effect of spiking analytes dissolved in organic solvents. The 
co-metabolic transformation of organic micropollutants (Plósz et al., 2010). A previous study found that the transformation of $\mathrm{COC}, \mathrm{BE}$ and $\mathrm{EME}$ seems to be unaffected by the readily biodegradable substrate content present in pre-clarified sewage (Plósz et al., 2013).

\section{Physical processes}

435 The overall findings for sorption to SPM in the bulk liquid (i.e., excluding biofilm on sewer walls) were similar in most studies. A detailed description of the methods applied to conduct these control experiments can be found in the supporting information. Sorption has been shown to play a limited role for COC, BE, AMP, METH, MDMA and COD, whereas some sorption was observed for EDDP, fentanyl and MTD (Baker and Kasprzyk-Hordern, 2011a, 2011b; Bisceglia and Lippa, 2014; Langford et al., 2011; Senta et al., 2014). Even more so, THC-COOH tends to sorb to SPM with increased sorption at low pH values (Khan and Nicell, 2012; Senta et al., 2014, 2013). No studies have specifically investigated the sorption of illicit drugs to the sewer biofilm. Substances that already tend to sorb to SPM in wastewater, e.g., THC-COOH, MTD and potentially LSD, may also sorb to sewer wall biofilms. Generally, since sorption is biomass-specific, extrapolating results from studies with SPM in wastewater to real systems with biofilm may not be adequate.

\section{Recommendations for future in-sewer experiments}

While it would be ideal to carry out in-sewer transformation studies in real sewers, there are several factors making full-scale experiments often (too) laborious to obtain accurate results, e.g., limited access to confined space, numerous confluents that would require monitoring and methods to experimentally account for unknown in- or exfiltration to close mass balances etc.,. Furthermore, due to varying environmental conditions that cannot be controlled during the experiments, several studies would be necessary. Based on the gaps identified in this review, we, therefore, propose the subsequent recommendations focusing on systematic laboratory and pilot-scale experiments to cover the wide range of realistic environmental conditions. This will facilitate interpretation and comparison of individual experiments, and allow to estimate the actual transformation potential in full-scale sewers.

\section{Biofilm}


Generally, if in-sewer experiments are performed in the laboratory, all sewer compartments should be considered, including biofilms growing on the sewer walls. Since recent in-sewer studies showed that biofilm could enhance transformation, future studies should consider realistic types and amounts of sewer biofilm. The result is an increase of the total biomass in the system that can influence transformation rates.

\section{Reproducibility and monitoring}

A major challenge of using real, fresh wastewater is the reproducibility of results. Its composition is likely to differ from experiment to experiment; therefore, a meticulous approach to the assessment of its composition and monitoring of the environmental conditions is necessary. We propose the following minimum set of parameters to be included: total suspended solids (TSS), volatile suspended solids (VSS) and the main process variables, dissolved oxygen, temperature, $\mathrm{pH}$, conductivity, dissolved inorganic sulfide/methane and soluble and total chemical oxygen demand.

\section{Spiking concentration levels}

The present illicit drug biomarker concentrations in grab samples or $24 \mathrm{~h}$ composite samples may be too low to conduct meaningful experiments. Degradation pathways and relevant processes must be considered carefully when deciding to spike substances, including using a mix of analytes in one experiment or multiple experiments with individual analytes. Further, the aim of the study (pathway investigation or stability monitoring) influences the decision on concentration levels, and willingness for sample preparation (effort for SPE). For kinetic stability studies, spiking may be necessary to guarantee levels of substances to be sufficiently above the Limit of Quantification (LOQ). In some cases, the use of deuterated or ${ }^{13} \mathrm{C}$-labelled compounds might be needed, since illicit drugs may already be present in the wastewater matrix. For pathway investigation studies, the spike of usually only one compound at high concentrations may be necessary. Ideally, the spike should be done without organic solvent.

\section{Controls}

To facilitate interpretation and meaningful comparison of experiments carried out at different points in time, in different laboratories and under different conditions, appropriate controls must be included. 
This can be done by analyzing substances with transformation behavior known from numerous studies, e.g., caffeine, nicotine (positive control) or carbamazepine (negative control). This may be helpful to compare different experiments and to confirm typical behavior in the setup.

Abiotic controls without active biomass and enzymes present are necessary to account for losses due to volatilization and abiotic transformations, such as chemical hydrolysis. We recommend to filter $(<1$ $\mu \mathrm{m})$ and potentially autoclave the abiotic control reactor content (Gulde et al., 2014; Helbling et al., 2010).

For certain substances, sorption controls are necessary to interpret the transformation data correctly and distinguish between losses due to biotransformation or sorption.

\section{Experimental design and reporting of results}

In most stability studies, the stability is represented as percentage decrease or increase from the initial concentration, determined from a sample at time $\mathrm{t}_{0}=0$ and $\mathrm{t}=\mathrm{t}_{\text {end }}$. In studies of micropollutant removal in wastewater treatment (pharmaceuticals, personal care products etc.), it is common to describe, if applicable, the exponential decrease in concentration over time with first-order kinetics (Schwarzenbach et al., 2003a). The resulting rate constants $\left(\mathrm{k}_{\mathrm{bio}}\right)$ are usually normalized to TSS or VSS, which facilitates comparison among different studies (i.e., different amount or activity of present biomass). We suggest performing in-sewer experiments over meaningful timeframes, which appears to be a relevant maximum hydraulic residence time in most sewer systems. To calculate rate constants, it is recommended to regularly collect samples, e.g., at the following points in time, related to the initial spike $\mathrm{t}\left(\mathrm{c}_{\text {background }}\right)=-5 \mathrm{~min}, \mathrm{t}\left(\mathrm{c}_{0}\right)=2-5 \mathrm{~min}$ after spike, $30 \mathrm{~min}, 1 \mathrm{~h}, 2 \mathrm{~h}, 4 \mathrm{~h}, 8 \mathrm{~h}, 16 \mathrm{~h}, 24 \mathrm{~h}$. The results should then be presented in conjunction with a suitable transformation model. For most substances, this may be (pseudo) first-order kinetics (Bisceglia and Lippa, 2014; Plósz et al., 2013).

\section{Sampling}

In kinetic studies, terminating the transformation processes at exact time points is crucial. After collecting the sample, the activity of the microorganisms can be stopped by adding a microorganism deactivating substance (see Controls); however, this will not stop abiotic processes. Guidelines suggest 
and extraction, with alternative centrifugation (and filtration) and separate extraction of the liquid and solid phase (OECD, 2008). Sample preservation and storage recommendations are provided in Section 5.

\section{Recommendations for preserving illicit drugs in wastewater samples}

515 In previous sections, stability of each specific compound has been discussed based on the available literature. However, most laboratories store and prepare samples for multi-class analysis. Therefore, in this section, recommendations on preserving illicit drugs are provided for multi-class methods. Key points associated with in-sample stability are: termination of transformation processes, filtration, preservation and long-term storage.

In general, samples should be filtered prior to storage. Filtration is mainly carried out to stop potential biotransformation and prevent clogging when performing SPE. A variety of membrane and glass fiber filters, with different pore sizes as low as $0.1 \mu \mathrm{m}$, are available and have been used (Baker and Kasprzyk-Hordern, 2011a; Östman et al., 2014). Smaller pore sizes are able to filter out suspended bacteria, but are more readily blocked, leading to an increase in preparation time (Chen et al., 2013). It is important to evaluate for possible losses of the analytes during the filtration process. The addition of mass-labelled internal standards prior to filtration and storage is recommended, in order to compensate for losses and transformation (Bijlsma et al., 2014; Castiglioni et al., 2013). Due to time constraints, wastewater samples are often frozen without previous filtration or the addition of labelled internal standards. In those cases, it is important to immediately add labelled internal standards after the sample has thawed. This can be justified by conducting freeze and thaw stability studies for the targeted analytes and accounting for the potential losses.

The most important aspects relating to in-sample stability is storage. Addition of preservatives, $\mathrm{pH}$ adjustment and storage at different temperatures has been studied in order to determine their impact. However, in a multi-class method, a compromise must be made for the optimal storage conditions. For example, lowering the $\mathrm{pH}$ increases the stability of several illicit drugs, except for THC-COOH (Baker and Kasprzyk-Hordern, 2011c; Chen et al., 2013; González-Mariño et al., 2010; Senta et al., 2014). If 
it is not possible to analyze the samples immediately, they can be stored upon arrival at $-20^{\circ} \mathrm{C}$ without any adjustments. Alternatively, samples could be extracted on SPE cartridges and stored at $-20^{\circ} \mathrm{C}$. Using these approaches, analytes are stable for up to 3 to 6 weeks, respectively (Baker and KasprzykHordern, 2011a; Chiaia et al., 2008; González-Mariño et al., 2010).

The stability of illicit drugs continues to be an issue during the subsequent sample preparation. Although this relates more to the analytical methodology and, therefore, is out of the scope of this review, it is worth mentioning that issues, such as the use of silanized glassware and evaporation temperature for reconstitution of sample extracts, should be addressed (Baker and Kasprzyk-Hordern, 2011a).

For in-sample stability (in a closed container), the following variable parameters are recommended to be measured at least at the beginning and end of the experiment: $\mathrm{pH}$, temperature, conductivity, TSS, VSS and soluble and total chemical oxygen demand. This will allow a more effective and reliable comparison of results among different experiments and conditions.

\section{Conclusions}

In wastewater-based epidemiology, the back-calculation currently used to estimate drug consumption at the population level does not account for potential in-sewer transformation of the targeted drug residues. This increases the uncertainty of estimates to an unknown degree.

Since most experiments were conducted with different grab wastewater samples of unknown composition, the occurring transformation processes in the studies might have varied. In addition, each study reported a different level of detail about these wastewater components and the experimental conditions, complicating the interpretation and generalization of compound behavior. Several illicit drugs, such as MDMA, KET and MDPV, seem to have a high stability in different wastewater studies at neutral $\mathrm{pH}$ and temperatures up to $20^{\circ} \mathrm{C}$. Also $\mathrm{BE}$ and $\mathrm{AMP}$ are most likely substances with high stability in those wastewater conditions; however, they may be formed as transformation products of other substances. Accordingly, the low stability of COC and 6-MAM seems to be well investigated. 
More research is needed for the drugs with variable behavior or few performed studies, such as THC-

$\mathrm{COOH}$, fentanyl, mephedrone and cathinones.

- The few studies to-date show that in-sewer transformation is compound-specific, influenced by the prevailing environmental conditions in sewers (temperature, sewer type). There is a lack of studies systematically investigating the influence of the different environmental conditions $(\mathrm{pH}$, suspended particulate matter, biofilm) on the transformation of illicit drugs.

- In order to compare different studies and environmental conditions, a reproducible experimental approach with quality controls for in-sewer transformation studies is needed. Therefore, this review recommends a best-practice approach for future in-sewer stability studies.

- Further, we summarize the best strategies to assure good in-sample stability of illicit drugs in the field of wastewater-based epidemiology. In multi-compound studies, most illicit drugs had a high stability at neutral $\mathrm{pH}$ and $-20^{\circ} \mathrm{C}$ for at least three weeks. Alternatively, acidification of the sample preserved most drugs, except for THC and metabolites.

\section{Acknowledgements}

Financial support by the European Union's Seventh Framework Program for research, technological development and demonstration SEWPROF (project no. 317205) is gratefully acknowledged. Phong Thai is partly supported by a UQ Postdoctoral Research Fellowship and a QUT VC Research Fellowship. Alexander van Nuijs acknowledges a post-doctoral fellowship from Flanders Funds for

Scientific Research (FWO). Lubertus Bijlsma acknowledges the financial support from Generalitat Valenciana (Group of Excellence Prometeo 2009/054, Prometeo II 2014/023; Collaborative Research on Environment and Food Safety ISIC/2012/016). Special thanks to Julianne McCall for proofreading the manuscript. 
Baker, D.R., Barron, L., Kasprzyk-Hordern, B., 2014. Illicit and pharmaceutical drug consumption estimated via wastewater analysis. Part A: Chemical analysis and drug use estimates. Sci. Total Environ. 487, 629-641. doi:10.1016/j.scitotenv.2013.11.107

Baker, D.R., Kasprzyk-Hordern, B., 2011a. Critical evaluation of methodology commonly used in sample collection, storage and preparation for the analysis of pharmaceuticals and illicit drugs in surface water and wastewater by solid phase extraction and liquid chromatography-mass spectrometry. J. Chromatogr. A 1218, 8036-8059. doi:10.1016/j.chroma.2011.09.012

Baker, D.R., Kasprzyk-Hordern, B., 2011b. Multi-residue determination of the sorption of illicit drugs and pharmaceuticals to wastewater suspended particulate matter using pressurised liquid extraction, solid phase extraction and liquid chromatography coupled with tandem mass spectrometry. J. Chromatogr. A 1218, 7901-7913. doi:10.1016/j.chroma.2011.08.092

Baker, D.R., Kasprzyk-Hordern, B., 2011c. Multi-residue analysis of drugs of abuse in wastewater and surface water by solid-phase extraction and liquid chromatography-positive electrospray ionisation tandem mass spectrometry. J. Chromatogr. A 1218, 1620-1631. doi:10.1016/j.chroma.2011.01.060

Baselt, R., 2008. Disposition of toxic drugs and chemicals in man. Biomed. Publ.

Bijlsma, L., Beltrán, E., Boix, C., Sancho, J. V., Hernández, F., 2014. Improvements in analytical methodology for the determination of frequently consumed illicit drugs in urban wastewater. Anal. Bioanal. Chem. 406, 4261-4272. doi:10.1007/s00216-014-7818-4

Bijlsma, L., Boix, C., Niessen, W.M. a, Ibáñez, M., Sancho, J. V, Hernández, F., 2013. Investigation of degradation products of cocaine and benzoylecgonine in the aquatic environment. Sci. Total Environ. 443, 200-208. doi:10.1016/j.scitotenv.2012.11.006

Bisceglia, K.J., 2007. Chapter 7 . Examination of Potential Sources of Error in Estimating Illicit Drug Consumption from Wastewater Measurements : Contributions of Drug Instability and Variability in Urinary Excretion.

Bisceglia, K.J.K., Lippa, K. a, 2014. Stability of cocaine and its metabolites in municipal wastewater the case for using metabolite consolidation to monitor cocaine utilization. Environ. Sci. Pollut. Res. 21, 4453-4460. doi:10.1007/s11356-013-2403-5

Boix, C., Ibáñez, M., Bijlsma, L., Sancho, J. V., Hernández, F., 2014. Investigation of cannabis biomarkers and transformation products in waters by liquid chromatography coupled to time of flight and triple quadrupole mass spectrometry. Chemosphere 99, 64-71. doi:10.1016/j.chemosphere.2013.10.007

Burgard, D.A., Banta-Green, C., Field, J.A., 2014. Working upstream: How far can you go with sewage-based drug epidemiology? Environ. Sci. Technol. 48, 1362-1368. doi:10.1021/es4044648

Burgard, D.A., Fuller, R., Becker, B., Ferrell, R., Dinglasan-Panlilio, M.J., 2013. Potential trends in Attention Deficit Hyperactivity Disorder (ADHD) drug use on a college campus: Wastewater analysis of amphetamine and ritalinic acid. Sci. Total Environ. 450-451, 242-249. doi:10.1016/j.scitotenv.2013.02.020 
Castiglioni, S., Bagnati, R., Melis, M., Panawennage, D., Chiarelli, P., Fanelli, R., Zuccato, E., 2011a. Identification of cocaine and its metabolites in urban wastewater and comparison with the human excretion profile in urine. Water Res. 45, 5141-5150. doi:10.1016/j.watres.2011.07.017

Castiglioni, S., Bijlsma, L., Covaci, A., Emke, E., Hernández, F., Reid, M., Ort, C., Thomas, K. V., Van Nuijs, A.L.N., De Voogt, P., Zuccato, E., 2013. Evaluation of uncertainties associated with the determination of community drug use through the measurement of sewage drug biomarkers. Environ. Sci. Technol. 47, 1452-1460. doi:10.1021/es302722f

Castiglioni, S., Borsotti, A., Senta, I., Zuccato, E., 2015. Wastewater Analysis to Monitor Spatial and Temporal Patterns of Use of Two Synthetic Recreational Drugs, Ketamine and Mephedrone, in Italy. Environ. Sci. Technol. 49, 5563-5570. doi:10.1021/es5060429

Castiglioni, S., Thomas, K. V., Kasprzyk-Hordern, B., Vandam, L., Griffiths, P., 2014. Testing wastewater to detect illicit drugs: State of the art, potential and research needs. Sci. Total Environ. 487, 613-620. doi:10.1016/j.scitotenv.2013.10.034

Castiglioni, S., Zuccato, E., Chiabrando, C., Fanelli, R., Bagnati, R., 2008. Mass spectrometric analysis of illicit drugs in wastewater and surface water. Mass Spectrom. Rev. 27, 378-394. doi:10.1002/mas.20168

Castiglioni, S., Zuccato, E., Crisci, E., Chiabrando, C., Fanelli, R., Bagnati, R., 2006. Identification and measurement of illicit drugs and their metabolites in urban wastewater by liquid chromatography-tandem mass spectrometry. Anal. Chem. 78, 8421-8429. doi:10.1021/ac061095b

Castiglioni, S., Zuccato, E., Fanelli, R., 2011b. Illicit Drugs in the Environment: Occurrence, Analysis, and Fate Using Mass Spectrometry, Illicit Drugs in the Environment: Occurrence, Analysis, and Fate Using Mass Spectrometry. John Wiley \& Sons. doi:10.1002/9781118000816

Chen, C., Kostakis, C., Irvine, R.J., Felgate, P.D., White, J.M., 2013. Evaluation of pre-analysis loss of dependent drugs in wastewater: Stability and binding assessments. Drug Test. Anal. 5, 716-721. doi:10.1002/dta.1428

Chiaia, A.C.A., Banta-Green, C., Field, J., 2008. Eliminating solid phase extraction with large-volume injection LC/MS/MS: analysis of illicit and legal drugs and human urine indicators in US wastewaters. Environ. Sci. Technol. 42, 8841-8848. doi:10.1021/es802309v

D’Ascenzo, G., Di Corcia, A., Gentili, A., Mancini, R., Mastropasqua, R., Nazzari, M., Samperi, R., 2003. Fate of natural estrogen conjugates in municipal sewage transport and treatment facilities. Sci. Total Environ. 302, 199-209. doi:10.1016/S0048-9697(02)00342-X

Evgenidou, E., 2015. Occurrence and removal of transformation products of ppcps and illicit drugs in wastewaters: a review. Sci. Total Environ. 505, 905-926. doi:10.1016/j.scitotenv.2014.10.021

Gheorghe, A., van Nuijs, A., Pecceu, B., Bervoets, L., Jorens, P.G., Blust, R., Neels, H., Covaci, A., 2008. Analysis of cocaine and its principal metabolites in waste and surface water using solidphase extraction and liquid chromatography-ion trap tandem mass spectrometry, in: Analytical and Bioanalytical Chemistry. pp. 1309-1319. doi:10.1007/s00216-007-1754-5

González-Mariño, I., Quintana, J.B., Rodríguez, I., Cela, R., 2010. Determination of drugs of abuse in water by solid-phase extraction, derivatisation and gas chromatography-ion trap-tandem mass spectrometry. J. Chromatogr. A 1217, 1748-1760. doi:10.1016/j.chroma.2010.01.046 
Gulde, R., Helbling, D.E., Scheidegger, A., Fenner, K., 2014. pH-dependent biotransformation of ionizable organic micropollutants in activated sludge. Environ. Sci. Technol. 48, 13760-8. doi:10.1021/es5037139

Helbling, D.D.E., Hollender, J., Kohler, H.-P.E.P.E., Singer, H., Fenner, K., 2010. High-throughput identification of microbial transformation products of organic micropollutants. Environ. Sci. Technol. 44, 6621-7. doi:10.1021/es100970m

Henze, M., Harremoës, P., La Cour Jansen, J., Arvin, E., 2002. Wastewater treatment: biological and chemical processes. Springer Science \& Business Media, New York, NY.

Heuett, N. V, Batchu, S.R., Gardinali, P.R., 2015a. Understanding the magnitude of emergent contaminant releases through target screening and metabolite identification using high resolution mass spectrometry: Illicit drugs in raw sewage influents. J. Hazard. Mater. 282, 41-50. doi:10.1016/j.jhazmat.2014.08.009

Heuett, N. V, Ramirez, C.E., Fernandez, A., Gardinali, P.R., 2015b. Analysis of drugs of abuse by online SPE-LC high resolution mass spectrometry : Communal assessment of consumption. Sci. Total Environ. 511, 319-330. doi:10.1016/j.scitotenv.2014.12.043

Hvitved-Jacobsen, T., Vollertsen, J., Nielsen, A.H., 2013. Sewer processes: microbial and chemical process engineering of sewer networks, second edi. ed. CRC Press, Boca Raton, FL.

Irvine, R.J., Kostakis, C., Felgate, P.D., Jaehne, E.J., Chen, C., White, J.M., 2011. Population drug use in Australia: A wastewater analysis. Forensic Sci. Int. 210, 69-73. doi:10.1016/j.forsciint.2011.01.037

Jelic, A., Rodriguez-Mozaz, S., Barceló, D., Gutierrez, O., 2014. Impact of in-sewer transformation on 43 pharmaceuticals in a pressurized sewer under anaerobic conditions. Water Res. 68, 98-108. doi:10.1016/j.watres.2014.09.033

Johnson, R.D., Botch-Jones, S.R., 2013. The Stability of Four Designer Drugs: MDPV, Mephedrone, BZP and TFMPP in Three Biological Matrices under Various Storage Conditions. J. Anal. Toxicol. 37, 51-55. doi:10.1093/jat/bks138

Karch, S., Jenkins, A., 2006. Drug abuse handbook, Chapter3 Pharamcokinetics: Drug Absorption, Distribution, and Elimination, 2nd editio. ed. CRC Press.

Khan, U., Nicell, J.A., 2012. Sewer epidemiology mass balances for assessing the illicit use of methamphetamine, amphetamine and tetrahydrocannabinol. Sci. Total Environ. 421-422, 144162. doi:10.1016/j.scitotenv.2012.01.020

Kinyua, J., Covaci, A., Maho, W., McCall, A.-K., Neels, H., van Nuijs, A.L.N., 2015. Sewage-based epidemiology in monitoring the use of new psychoactive substances: Validation and application of an analytical method using LC-MS/MS. Drug Test. Anal. 7, 812-818. doi:10.1002/dta.1777

700 Kraemer, T., Maurer, H.H., 2002. Toxicokinetics of amphetamines: metabolism and toxicokinetic data of designer drugs, amphetamine, methamphetamine, and their N-alkyl derivatives. Ther. Drug Monit. 24, 277-289. doi:10.1097/00007691-200204000-00009

Lai, F.Y., Anuj, S., Bruno, R., Carter, S., Gartner, C., Hall, W., Kirkbride, K.P., Mueller, J.F., Brien, J.W.O., Prichard, J., Thai, P.K., Ort, C., 2015. Systematic and Day-to-Day E ff ects of ChemicalDerived Population Estimates on Wastewater-Based Drug Epidemiology. Environ. Sci. Technol. 49, 999-1008. doi:10.1021/es503474d 
Lai, F.Y., Bruno, R., Hall, W., Gartner, C., Ort, C., Kirkbride, P., Prichard, J., Thai, P.K., Carter, S., Mueller, J.F., 2013. Profiles of illicit drug use during annual key holiday and control periods in Australia: Wastewater analysis in an urban, a semi-rural and a vacation area. Addiction 108, 556-565. doi:10.1111/add.12006

Lai, F.Y., Ort, C., Gartner, C., Carter, S., Prichard, J., Kirkbride, P., Bruno, R., Hall, W., Eaglesham, G., Mueller, J.F., 2011. Refining the estimation of illicit drug consumptions from wastewater analysis: co-analysis of prescription pharmaceuticals and uncertainty assessment. Water Res. 45, 4437-4448. doi:10.1016/j.watres.2011.05.042

Langford, K.H., Reid, M., Thomas, K. V, 2011. Multi-residue screening of prioritised human pharmaceuticals, illicit drugs and bactericides in sediments and sludge. J. Environ. Monit. 13, 2284-2291. doi:10.1039/c1em10260e

Mardal, M., Meyer, M.R., 2014. Department of Experimental and Clinical Toxicology, Institute of Experimental and Clinical Pharmacology and Toxicology, Saarland University, Homburg (Saar), Germany. Sci. Total Environ. 493, 1-18. doi:10.1016/j.scitotenv.2014.06.016

Maurer, M., Escher, B.I., Richle, P., Schaffner, C., Alder, A.C., 2007. Elimination of beta-blockers in sewage treatment plants. Water Res. 41, 1614-1622. doi:10.1016/j.watres.2007.01.004

Metcalfe, C., Tindale, K., Li, H., Rodayan, A., Yargeau, V., 2010. Illicit drugs in Canadian municipal wastewater and estimates of community drug use. Environ. Pollut. 158, 3179-3185. doi:10.1016/j.envpol.2010.07.002

O’Brien, J., Thai, P.K., Eaglesham, G., Ort, C., Scheidegger, A., Carter, S., Lai, F.Y., Mueller, J.F., 2014. A Model to Estimate the Population Contributing to the Wastewater Using Samples Collected on Census Day. Environ. Sci. Technol. 48, 517-525. doi:10.1021/es403251g

OECD, 2008. Guidelines for the Testing of Chemicals. OECD 314: Simulation tests to assess the biodegradability of chemicals discharged in wastewater. Paris.

Ort, C., Gujer, W., 2008. Sorption and high dynamics of micropollutants in sewers.

Ort, C., Lawrence, M.G., Reungoat, J., Mueller, J.F., 2010. Sampling for PPCPs in wastewater systems: comparison of different sampling modes and optimization strategies. Environ. Sci. Technol. 44, 6289-96. doi:10.1021/es100778d

735 Ort, C., van Nuijs, A.L.N., Berset, J.D., Bijlsma, L., Castiglioni, S., Covaci, A., de Voogt, P., Emke, E., Fatta-Kassinos, D., Griffiths, P., Hernández, F., González-Mariño, I., Grabic, R., KasprzykHordern, B., Mastroianni, N., Meierjohann, A., Nefau, T., Östman, M., Pico, Y., Racamonde, I., Reid, M., Slobodnik, J., Terzic, S., Thomaidis, N., Thomas, K. V, 2014. Spatial differences and temporal changes in illicit drug use in Europe quantified by wastewater analysis. Addiction 109, 1338-1352. doi:10.1111/add.12570

Östman, M., Fick, J., Näsström, E., Lindberg, R.H., 2014. A snapshot of illicit drug use in Sweden acquired through sewage water analysis. Sci. Total Environ. 472, 862-871. doi:10.1016/j.scitotenv.2013.11.081

Plósz, B.G., Leknes, H., Thomas, K. V, 2010. Impacts of competitive inhibition, parent compound formation and partitioning behavior on the removal of antibiotics in municipal wastewater treatment. Environ. Sci. Technol. 44, 734-742. doi:10.1021/es902264w 
Plósz, B.G., Reid, M.J., Borup, M., Langford, K.H., Thomas, K. V., 2013. Biotransformation kinetics and sorption of cocaine and its metabolites and the factors influencing their estimation in wastewater. Water Res. 47, 2129-2140. doi:10.1016/j.watres.2012.12.034

Postigo, C., Sirtori, C., Oller, I., Malato, S., Maldonado, M.I., López de Alda, M., Barceló, D., 2011a. Solar transformation and photocatalytic treatment of cocaine in water: Kinetics, characterization of major intermediate products and toxicity evaluation. Appl. Catal. B Environ. 104, 37-48. doi:10.1016/j.apcatb.2011.02.030

Postigo, C., Sirtori, C., Oller, I., Malato, S., Maldonado, M.I., López de Alda, M., Barceló, D., 2011b. Photolytic and photocatalytic transformation of methadone in aqueous solutions under solar irradiation: Kinetics, characterization of major intermediate products and toxicity evaluation. Water Res. 45, 4815-4826. doi:10.1016/j.watres.2011.06.027

Reid, M.J., Baz-Lomba, J.A., Ryu, Y., Thomas, K. V, 2014. Using biomarkers in wastewater to monitor community drug use: A conceptual approach for dealing with new psychoactive substances. Sci. Total Environ. 487, 651-658. doi:10.1016/j.scitotenv.2013.12.057

Rodayan, A., Segura, P.A., Yargeau, V., 2014. Ozonation of wastewater: Removal and transformation products of drugs of abuse. Sci. Total Environ. 487, 763-770. doi:10.1016/j.scitotenv.2013.11.023

Rosa Boleda, M., Huerta-Fontela, M., Ventura, F., Galceran, M.T., 2011. Evaluation of the presence of drugs of abuse in tap waters. Chemosphere 84, 1601-1607. doi:10.1016/j.chemosphere.2011.05.033

Schwarzenbach, R.P., Gschwend, P.M., Imboden, D.M., 2003a. Transformation Processes, in: Environmental Organic Chemistry. John Wiley \& Sons, Inc., pp. 459-460. doi:10.1002/0471649643

770 Schwarzenbach, R.P., Gschwend, P.M., Imboden, D.M., 2003b. General Topic and Overview, in: Environmental Organic Chemistry. John Wiley \& Sons, Inc., pp. 3-12. doi:10.1002/0471649643

Senta, I., Krizman, I., Ahel, M., Terzic, S., 2013. Integrated procedure for multiresidue analysis of dissolved and particulate drugs in municipal wastewater by liquid chromatography-tandem mass spectrometry. Anal. Bioanal. Chem. 405, 3255-3268. doi:10.1007/s00216-013-6720-9

775 Senta, I., Krizman, I., Ahel, M., Terzic, S., 2014. Assessment of stability of drug biomarkers in municipal wastewater as a factor influencing the estimation of drug consumption using sewage epidemiology. Sci. Total Environ. 487, 659-665. doi:10.1016/j.scitotenv.2013.12.054

Sharma, K., Ganigue, R., Yuan, Z., 2013. PH dynamics in sewers and its modeling. Water Res. 47, 6086-6096. doi:10.1016/j.watres.2013.07.027

780 Siegrist, H., Joss, A., 2012. Review on the fate of organic micropollutants in wastewater treatment and water reuse with membranes. Water Sci. Technol. 66, 1369-1376. doi:10.2166/wst.2012.285

Subedi, B., Kannan, K., 2014. Mass loading and removal of select illicit drugs in two wastewater treatment plants in New York State and estimation of illicit drug usage in communities through wastewater analysis. Environ. Sci. Technol. 48, 6661-6670. doi:10.1021/es501709a

Tchobanoglous, G., Burton, F., 1991. Wastewater Engineering: treatment, disposal and reuse, 3rd ed. Metcalf \& Eddy McGraw-Hill, New York, NY. 
Ternes, T.A., Joss, A., 2006. Human Pharmaceuticals, Hormones and Fragrances, IWA Publishing , London, UK. IWA.

Thai, P.K., Jiang, G., Gernjak, W., Yuan, Z., Lai, F.Y., Mueller, J.F., 2014. Effects of sewer conditions on the degradation of selected illicit drug residues in wastewater. Water Res. 48, 538 547. doi:10.1016/j.watres.2013.10.019

Thomas, K. V, Bijlsma, L., Castiglioni, S., Covaci, A., Emke, E., Grabic, R., Hernández, F., Karolak, S., Kasprzyk-Hordern, B., Lindberg, R.H., Lopez de Alda, M., Meierjohann, A., Ort, C., Pico, Y., Quintana, J.B., Reid, M., Rieckermann, J., Terzic, S., van Nuijs, A.L.N., de Voogt, P., 2012. Comparing illicit drug use in 19 European cities through sewage analysis. Sci. Total Environ. 432, 432-439. doi:10.1016/j.scitotenv.2012.06.069

United Nations Office of Drug and Crime, 2014. World Drug Report 2014. Vienna.

Van Nuijs, A.L.N., Abdellati, K., Bervoets, L., Blust, R., Jorens, P.G., Neels, H., Covaci, A., 2012. The stability of illicit drugs and metabolites in wastewater, an important issue for sewage epidemiology? J. Hazard. Mater. 239-240, 19-23. doi:10.1016/j.jhazmat.2012.04.030

Van Nuijs, A.L.N., Castiglioni, S., Tarcomnicu, I., Postigo, C., de Alda, M.L., Neels, H., Zuccato, E., Barcelo, D., Covaci, A., 2011a. Illicit drug consumption estimations derived from wastewater analysis: A critical review. Sci. Total Environ. doi:10.1016/j.scitotenv.2010.05.030

Van Nuijs, A.L.N., Mougel, J.-F., Tarcomnicu, I., Bervoets, L., Blust, R., Jorens, P.G., Neels, H., Covaci, A., 2011b. Sewage epidemiology--a real-time approach to estimate the consumption of illicit drugs in Brussels, Belgium. Environ. Int. 37, 612-621. doi:10.1016/j.envint.2010.12.006

Van Nuijs, A.L.N., Pecceu, B., Theunis, L., Dubois, N., Charlier, C., Jorens, P.G., Bervoets, L., Blust, R., Neels, H., Covaci, A., 2009a. Spatial and temporal variations in the occurrence of cocaine and benzoylecgonine in waste- and surface water from Belgium and removal during wastewater treatment. Water Res. 43, 1341-1349. doi:10.1016/j.watres.2008.12.020

Van Nuijs, A.L.N., Pecceu, B., Theunis, L., Dubois, N., Charlier, C., Jorens, P.G., Bervoets, L., Blust, R., Neels, H., Covaci, A., 2009b. Cocaine and metabolites in waste and surface water across Belgium. Environ. Pollut. 157, 123-129. doi:10.1016/j.envpol.2008.07.020

Vazquez-Roig, P., Blasco, C., Picó, Y., 2013. Advances in the analysis of legal and illegal drugs in the aquatic environment. Trends Anal. Chem. doi:10.1016/j.trac.2013.04.008

Warner, A., Norman, A.B., 2000. Mechanisma of cocaine Hydrolysis and metabolism in vitro and in vivo: a clarification. Ther. Drug Monit. 22, 266-270. doi:Doi 10.1097/00007691-20000600000006

Wick, A., Wagner, M., Ternes, T.A., 2011. Elucidation of the transformation pathway of the opium alkaloid codeine in biological wastewater treatment. Environ. Sci. Technol. 45, 3374-3385. doi:10.1021/es103489x

Zuccato, E., Chiabrando, C., Castiglioni, S., Calamari, D., Bagnati, R., Schiarea, S., Fanelli, R., 2005. Cocaine in surface waters: a new evidence-based tool to monitor community drug abuse. Environ. Health 4, 14. doi:10.1186/1476-069X-4-14 